ESAIM: M2AN 46 (2012) 681-707

DOI: $10.1051 / \mathrm{m} 2 \mathrm{an} / 2011047$
ESAIM: Mathematical Modelling and Numerical Analysis

www.esaim-m2an.org

\title{
IMPLICIT-EXPLICIT RUNGE-KUTTA SCHEMES AND FINITE ELEMENTS WITH SYMMETRIC STABILIZATION FOR ADVECTION-DIFFUSION EQUATIONS
}

\author{
Erik Burman ${ }^{1}$ AND Alexandre Ern ${ }^{2}$
}

\begin{abstract}
We analyze a two-stage implicit-explicit Runge-Kutta scheme for time discretization of advection-diffusion equations. Space discretization uses continuous, piecewise affine finite elements with interelement gradient jump penalty; discontinuous Galerkin methods can be considered as well. The advective and stabilization operators are treated explicitly, whereas the diffusion operator is treated implicitly. Our analysis hinges on $L^{2}$-energy estimates on discrete functions in physical space. Our main results are stability and quasi-optimal error estimates for smooth solutions under a standard hyperbolic CFL restriction on the time step, both in the advection-dominated and in the diffusiondominated regimes. The theory is illustrated by numerical examples.
\end{abstract}

Mathematics Subject Classification. 5M12, 65M15, 65M60.

Received October 28, 2010. Revised May 31, 2011.

Published online February 3, 2012.

\section{INTRODUCTION}

We consider the transient advection-diffusion equation

$$
\begin{aligned}
\partial_{t} u+B u+A u & =f \text { in } \Omega \times\left(0, t_{\mathrm{F}}\right), \\
u & =0 \text { on } \partial \Omega \times\left(0, t_{\mathrm{F}}\right), \\
u(\cdot, t=0) & =u_{0} \text { in } \Omega,
\end{aligned}
$$

where $\Omega$ is a polyhedron in $\mathbb{R}^{d}$ with boundary $\partial \Omega, B u:=\beta \cdot \nabla u, A u:=-\mu \Delta u, t_{\mathrm{F}}>0$ a finite time, $\beta$ a divergence-free velocity field, $\mu>0$ the diffusion coefficient, $f$ the source term, and $u_{0}$ the initial datum. Extensions of the present analysis to advection fields with nonzero divergence and inclusion of non-stiff zeroorder terms is straightforward; accounting for smoothly variable diffusion coefficient is also feasible.

In the stationary case, it is well-known that the standard Galerkin finite element method has poor stability properties in the advection-dominated regime, resulting in suboptimal convergence for smooth solutions

\footnotetext{
Keywords and phrases. Stabilized finite elements, stability, error bounds, implicit-explicit Runge-Kutta schemes, unsteady convection-diffusion.

1 Department of Mathematics, University of Sussex, Brighton, BN1 9QH, UK. E.N.Burman@sussex.ac.uk

2 Université Paris-Est, CERMICS, École des Ponts, 77455 Marne la Vallée Cedex 2, France. ern@cermics.enpc.fr
} 
and spurious oscillations when approximating solutions with sharp layers. Different approaches have been proposed to improve this behavior, such as the streamline upwind Petrov-Galerkin method (SUPG) [4, 23] and standard Galerkin methods with symmetric stabilization in various flavors, e.g., discontinuous Galerkin (DG) $[16,18,24,25]$, subgrid viscosity [19,20], orthogonal subscale stabilization [14], local projection stabilization $[3,28]$, and continuous interior penalty on interelement normal gradient jumps (CIP) [5,10]. All these methods lead to similar $L^{2}$-norm error estimates for smooth solutions, resulting in the loss of half a power of $h$ in the advection-dominated regime (compared to a full power in the unstabilized case). For solutions with sharp layers, it has been proven for SUPG [23], DG [21], and CIP [12] that quasi-optimal convergence is retained away from layers, hence prohibiting the global spreading of spurious oscillations.

In the transient case, DG-based time discretization has been the favored alternative for SUPG [23], whereas Runge-Kutta (RK) methods have been popular for time discretization combined with DG in space [13]. For symmetric stabilizations in general, standard A-stable finite difference methods in time have been shown to be stable and optimally convergent $[9,14,17,20]$. Similar results for SUPG and the transient advection-diffusion equation are very recent $[6,11]$. The implicit time stepping by A-stable methods leads to a nonsymmetric matrix to be inverted at each time step. Moreover, treating nonlinear transport operators with such methods or incorporating nonlinear slope limiters can be quite demanding computationally. Ideally, one would like to treat the advective and stabilization operators explicitly and the diffusive operator implicitly. A suitable class of methods is that of implicit-explicit (IMEX) RK methods. The application of IMEX methods to partial differential equations (PDEs) was first analyzed in [15], and IMEX RK methods were first proposed in $[1,2]$. From a computational viewpoint, IMEX RK methods only require symmetric systems to be solved at each time step, and the stencil of the corresponding matrix is that of the diffusion operator. Moreover, nonlinear transport operators and nonlinear slope limiters can be treated explicitly.

Although a substantial amount of literature exists on IMEX RK methods, deriving stability and error estimates for stabilized finite elements combined with IMEX RK time discretization remains, to the authors' knowledge, an open issue. In particular, we aim at an analysis that is valid in all flow regimes, that is, either advection-dominated or diffusion-dominated. Following the seminal work of Levy and Tadmor [26], the present analysis relies on $L^{2}$-energy estimates, that is, we work directly with discrete functions in the physical space. In other words, we account for the full geometric structure of eigenvectors, instead of the more classical approach using only scalar eigenvalue arguments which may be misleading in the context of nonnormal operators.

Concerning IMEX RK schemes, a first important issue uncovered herein is that the analysis of the truncation error in time by means of Butcher tables is not sufficient in the context of PDEs. In particular, this error involves the partial differential operators $A$ and $B$ acting on suitable functions associated with the intermediate stages of the scheme. In the IMEX scheme, bounding (high-order) derivatives of these functions is not straightforward and, in particular, requires a careful study of the role played by boundary conditions. A second important issue is that the explicit part of the RK scheme is anti-dissipative, that is, it produces energy, so that this energy production must be controlled by the stability induced by space discretization. In the context of finite element methods with symmetric stabilization, explicit (second- and third-order) RK methods were analyzed in [8], in particular for the pure advection equation, leading to stability and error estimates for smooth solutions. The presence of the diffusion operator poses additional difficulties to be tackled herein.

The two-stage IMEX RK scheme we consider for time discretization is the so-called SSP2 $(2,2,2) L$-stable scheme proposed in [27] for hyperbolic systems with stiff relaxation terms and no sources. This scheme combines an explicit two-stage RK scheme for the transport operator together with a diagonally implicit, two-stage RK scheme for the stiff relaxation terms. Moreover, this scheme is formulated in terms of a parameter $\gamma$, and the value $\gamma=\gamma_{*}:=1-\frac{1}{\sqrt{2}} \simeq 0.293$ is considered in [27]. Herein, we apply and analyze, for the first time, this scheme in the context of advection-diffusion equations. Space discretization is performed using continuous, piecewise affine finite elements with CIP as a specific example of symmetric stabilization; DG methods can be used as well, as discussed toward the end of the manuscript. We treat the advection and stabilization operators explicitly and the diffusion operator implicitly. 
Our main results are stability and error estimates for smooth solutions in all flow regimes. These results are formulated in terms of the Courant and Péclet numbers defined as

$$
\text { Co }:=\frac{\sigma \tau}{h}, \quad \mathrm{Pe}:=\frac{\sigma h}{\mu},
$$

where $\sigma:=\|\beta\|_{L^{\infty}(\Omega)}$ is the reference velocity, $h$ the mesh size, and $\tau$ the time step. For simplicity, the time step is taken to be constant, and we use a single Péclet number for the whole domain. In all flow regimes, we assume a hyperbolic type CFL restriction on the time step of the form Co $\leq \varrho$ with $\varrho$ independent of the mesh size $h$, the time step $\tau$, and the problem data. Furthermore, the analysis of the truncation error in time requires the technical assumptions that the normal component of $\beta$ and the source term $f$ vanish on $\partial \Omega$ and that elliptic regularity holds for the Laplace operator. In the advection-dominated regime $(\mathrm{Pe} \geq 1)$, stability and convergence are achieved for $\gamma \in\left(0, \frac{1}{2}\right)$; to fix the ideas, we take $\gamma \in\left[\frac{1}{5}, \frac{2}{5}\right]$ (the actual value of $\gamma$ influences only the numerical bound on the Courant number). Our main convergence result (Thm. 4.8 and Prop. 4.9) takes the form

$$
\left\|u\left(t_{\mathrm{F}}\right)-u_{h}^{N}\right\|_{L^{2}(\Omega)}+\left(\tau \sum_{n=1}^{N} \mu\left\|\nabla\left(u\left(t^{n}\right)-u_{h}^{n}\right)\right\|_{L^{2}(\Omega)^{d}}^{2}\right)^{1 / 2} \lesssim \tau^{3 / 2}+\sigma^{1 / 2} h^{3 / 2} .
$$

The estimate for the space error is quasi-optimal (1/2-suboptimal), similarly to the steady case. The estimate for the time error is also quasi-optimal (1/2-suboptimal considering that a two-stage IMEX RK scheme is used, and this is a consequence of the need to bound space derivatives when estimating the truncation error in time). Owing to the CFL restriction on the time step, this estimate is actually sufficient to equilibrate space and time errors. In the diffusion-dominated regime $(\mathrm{Pe} \leq 1)$, stability and convergence are achieved for $\gamma$ in a sufficiently small neighborhood of $\gamma_{*}$. In addition to the bound on the Courant number (which becomes trivial in the purediffusion limit), the time step is restricted by the bound $\tau \leq\left(t_{*} / \mu\right)^{1 / 2} h$ where $t_{*}$ is a reference time defined in Section 2.1. Our main convergence result (Thm. 4.16) takes the form

$$
\left(\tau \sum_{n=1}^{N} \mu\left\|\nabla\left(u\left(t^{n}\right)-u_{h}^{n}\right)\right\|_{L^{2}(\Omega)^{d}}^{2}\right)^{1 / 2} \lesssim \tau+\mu^{1 / 2} h
$$

The estimate on the space error is optimal, while the estimate on the time error is 1-suboptimal, but, again, owing to the CFL restriction, it is actually sufficient to equilibrate space and time errors. Finally, still in the diffusion-dominated regime, we prove that (Prop. 4.17)

$$
\left\|u\left(t_{\mathrm{F}}\right)-u_{h}^{N}\right\|_{L^{2}(\Omega)} \lesssim \tau^{3 / 2}+\sigma^{1 / 2} h^{3 / 2}+\mu^{-1 / 2} h^{2} .
$$

This estimate is $1 / 2$-suboptimal in time and in space, but, as the other estimates, equilibrates both errors owing to the CFL restriction. Moreover, as $\sigma \rightarrow 0$, that is, in the pure diffusion limit, second-order convergence is recovered in $h$. Finally, we observe that under an additional assumption on $\beta$ at the boundary, the convergence order in time of all the above estimates can be improved by a factor $\tau^{1 / 2}$; see Remark 3.7.

The material is organized as follows. Section 2 states the basic assumptions, presents the setting for the space and time discretization, and introduces the truncation error in time together with the error equations. Section 3 is devoted to the analysis of the truncation error and the approximation error in space. Section 4 contains the stability and error analysis, while Section 5 discusses extensions to other space discretization schemes. Section 6 presents numerical results. In what follows, we often abbreviate $a \lesssim b$ the inequality $a \leq C b$ for positive $C$ independent of the mesh size $h$, the time step $\tau$, and the problem data. We only keep track of constants if they are to be used later in thresholds for the Courant number. 


\section{The SETTing}

In this section, we specify the basic assumptions for the time evolution problem (1.1) and the discretization parameters. We also present the stabilized finite element method for space discretization together with the twostage IMEX RK scheme for time discretization. Then, we express the truncation error in time using suitable intermediate functions associated with the intermediate stages of the IMEX RK scheme, and we derive the error equation. Finally, we collect important stability and boundedness properties of the discrete operators used for space discretization.

\subsection{Basic assumptions}

Let $L:=L^{2}(\Omega), L^{d}:=L^{2}(\Omega)^{d}$, and set $V:=H^{2}(\Omega) \cap H_{0}^{1}(\Omega)$. We assume that the exact solution $u$ and the source term $f$ are such that

$$
\begin{aligned}
& u \in C^{0}\left(\left[0, t_{\mathrm{F}}\right] ; H^{4}(\Omega) \cap H_{0}^{1}(\Omega)\right) \cap C^{1}\left(\left[0, t_{\mathrm{F}}\right] ; H^{3}(\Omega)\right) \cap C^{3}\left(\left[0, t_{\mathrm{F}}\right] ; L\right), \\
& f \in C^{0}\left(\left[0, t_{\mathrm{F}}\right] ; H^{2}(\Omega) \cap H_{0}^{1}(\Omega)\right) \cap C^{2}\left(\left[0, t_{\mathrm{F}}\right] ; L\right),
\end{aligned}
$$

and we observe that (2.1b) means, in particular, that $\left.f\right|_{\partial \Omega}=0$. We assume that the domain $\Omega$ is convex so that elliptic regularity holds true for the Laplace operator with homogeneous Dirichlet boundary conditions. Finally, we assume that $\beta$ is in the Sobolev space $\left[W^{1, \infty}(\Omega)\right]^{d}$, so that $\beta$ is bounded and has bounded derivatives, and that the normal component of $\beta$ vanishes at the boundary, that is, $\left.\nu \cdot \beta\right|_{\partial \Omega}=0$ where $\nu$ denotes the unit outward normal to $\Omega$. For later use, we set $\sigma_{1}:=\|\nabla \beta\|_{\left[L^{\infty}(\Omega)\right]^{d, d}}$ and observe that $\sigma_{1}^{-1}$ can be interpreted as a time scale. We also consider the reference time $t_{*}:=\min \left(\sigma_{1}^{-1}, t_{\mathrm{F}}\right)$.

An important consequence of the fact that the normal component of $\beta$ and the source term $f$ vanish at the boundary is the following.

Proposition 2.1 (boundary value of $B u(t)$ and $A u(t))$. For all $t \in\left[0, t_{\mathrm{F}}\right]$,

$$
\left.B u(t)\right|_{\partial \Omega}=\left.A u(t)\right|_{\partial \Omega}=0 .
$$

Proof. The fact that $\left.B u(t)\right|_{\partial \Omega}=0$ results from $\beta$ having zero normal component on $\partial \Omega$ and $u$ vanishing on $\partial \Omega$. The fact that $\left.A u(t)\right|_{\partial \Omega}=0$ then results from the evolution equation since $\left.f(t)\right|_{\partial \Omega}=\left.\partial_{t} u(t)\right|_{\partial \Omega}=0$.

Concerning the discretization parameters, we always assume to fix the ideas that Co $\leq 1$; bounds on the Courant number with different constants will be introduced later. We also assume the following mild reverseparabolic CFL inequality

$$
h^{2} \lesssim \bar{\mu} \tau,
$$

where $\bar{\mu}:=\max \left(\mu, \sigma^{2} t_{*}\right)$. Condition $(2.3)$ is not needed in the stability analysis, but only to derive a bound on the truncation error in time of order $\tau^{3 / 2}$, instead of $\tau$; see Lemma 3.6. Finally, we make the mild assumption that the mesh size and the time step resolve the spatial variations of the advection velocity, that is,

$$
\sigma_{1} h \leq \sigma, \quad \sigma_{1} \tau \leq 1,
$$

and observe that the second bound implies $\tau \leq t_{*}$ since $\tau \leq t_{\mathrm{F}}$ as well.

\subsection{Space discretization}

Let $\left\{\mathcal{T}_{h}\right\}_{h>0}$ be a family of affine, simplicial meshes of $\Omega$. We assume that the meshes are kept fixed in time and that the family $\left\{\mathcal{T}_{h}\right\}_{h>0}$ is quasi-uniform. It is also possible to work with shape-regular mesh families. In this case, as usual, the space scale in the CFL condition is no longer $h$, but the smallest element diameter in the mesh. Mesh faces are collected in the set $\mathcal{F}_{h}$ which is split into the set of interior faces, $\mathcal{F}_{h}^{\text {int }}$, and boundary faces, $\mathcal{F}_{h}^{\text {ext }}$. For a smooth enough function $v$ that is possibly double-valued at $F \in \mathcal{F}_{h}^{\text {int }}$ with $F=\partial T^{-} \cap \partial T^{+}$, 
we define its jump at $F$ as $\llbracket v \rrbracket:=\left.v\right|_{T^{-}}-\left.v\right|_{T^{+}}$, and we fix the unit normal vector to $F$, denoted by $\nu_{F}$, as pointing from $T^{-}$to $T^{+}$. The arbitrariness in the sign of $\llbracket v \rrbracket$ is irrelevant in what follows.

Let $V_{h}$ be the finite element space spanned by continuous and piecewise affine functions. Set $V(h):=H^{2}(\Omega)+$ $V_{h}$. The space semi-discretized formulation can be written as follows: for all $t \in\left[0, t_{\mathrm{F}}\right]$, find $u_{h}(t) \in V_{h}$ such that

$$
\partial_{t} u_{h}(t)+B_{h} u_{h}(t)+A_{h} u_{h}(t)=f_{h}(t),
$$

with initial condition $u_{h}(0)=\pi_{h} u_{0}$ and source term $f_{h}:=\pi_{h} f$, where $\pi_{h}$ denotes the $L$-orthogonal projection onto $V_{h}$. The discrete linear operators $B_{h}: V(h) \rightarrow V_{h}$ and $A_{h}: V(h) \rightarrow V_{h}$ are such that for all $\left(z, w_{h}\right) \in$ $V(h) \times V_{h}$,

$$
\begin{aligned}
\left(B_{h} z, w_{h}\right)_{L} & :=\left(\beta \cdot \nabla z, w_{h}\right)_{L}+\sum_{F \in \mathcal{F}_{h}^{\text {int }}} S_{\text {cip }} h_{F}^{2}\left(\left|\nu_{F} \cdot \beta\right| \nu_{F} \cdot \llbracket \nabla z \rrbracket, \nu_{F} \cdot \llbracket \nabla w_{h} \rrbracket\right)_{L, F}, \\
\left(A_{h} z, w_{h}\right)_{L} & :=\left(\mu \nabla z, \nabla w_{h}\right)_{L^{d}}-\left(\mu(\nu \cdot \nabla z), w_{h}\right)_{L, \partial \Omega}-\left(\mu z, \nu \cdot \nabla w_{h}\right)_{L, \partial \Omega}+S_{\mathrm{bc}} h^{-1}\left(\mu z, w_{h}\right)_{L, \partial \Omega} .
\end{aligned}
$$

Here, $(\cdot, \cdot)_{L}$ denotes the $L^{2}(\Omega)$-inner product (with associated norm $\left.\|\cdot\|_{L}\right)$ and $(\cdot, \cdot)_{L^{d}}$ the $\left[L^{2}(\Omega)\right]^{d}$-inner product (with associated norm $\|\cdot\|_{L^{d}}$ ), while, for a subset $\omega \subset \Omega$ (a mesh face or a collection thereof), $(\cdot, \cdot)_{L, \omega}$ denotes the corresponding $L^{2}(\omega)$-inner product. We observe that the homogeneous Dirichlet boundary condition is weakly enforced in $A_{h}$ (and that the additional boundary term $\sum_{F \in \mathcal{F}_{h}^{\text {ext }} \cap \partial \Omega^{-}}\left(|\nu \cdot \beta| z, v_{h}\right)_{L, F}$, where $\partial \Omega^{-}$denotes the inflow boundary, has been discarded from $B_{h}$ since we assume $\left.\nu \cdot \beta\right|_{\partial \Omega}=0$ ). Moreover, the user-dependent parameter $S_{\text {cip }}$ is positive, while the user-dependent parameter $S_{\mathrm{bc}}$ is sufficiently large (see Sect. 2.6).

The discrete linear operators $A_{h}$ and $B_{h}$ satisfy important stability and boundedness properties collected in Section 2.6. For the time being, we record the following consistency property: for all $v \in V$,

$$
B_{h} v=\pi_{h}(B v), \quad A_{h} v=\pi_{h}(A v) .
$$

\subsection{Time discretization}

For all $0 \leq n \leq N$ with $N:=\left\lfloor t_{\mathrm{F}} / \tau\right\rfloor$, a superscript $n$ indicates the value of a function at the discrete time $n \tau$, and for all $0 \leq n \leq N-1$, we set $I_{n}:=(n \tau,(n+1) \tau]$. For a real parameter $\gamma \in\left(0, \frac{1}{2}\right)$, we consider the following time discretization scheme:

$$
\begin{aligned}
v_{h}^{n} & =u_{h}^{n}-\gamma \tau A_{h} v_{h}^{n}+\gamma \tau f_{h}^{n}, \\
w_{h}^{n} & =u_{h}^{n}-\tau B_{h} v_{h}^{n}-(1-2 \gamma) \tau A_{h} v_{h}^{n}-\gamma \tau A_{h} w_{h}^{n}+(1-\gamma) \tau f_{h}^{n}, \\
u_{h}^{n+1} & =u_{h}^{n}-\frac{1}{2} \tau B_{h}\left(v_{h}^{n}+w_{h}^{n}\right)-\frac{1}{2} \tau A_{h}\left(v_{h}^{n}+w_{h}^{n}\right)+\tau f_{h}^{n+\frac{1}{2}} .
\end{aligned}
$$

Here, $f_{h}^{n+\frac{1}{2}}:=\pi_{h} f\left(\left(n+\frac{1}{2}\right) \tau\right)$ can be replaced by any second-order approximation in time, e.g., $\frac{1}{2}\left(f_{h}^{n}+f_{h}^{n+1}\right)$. We observe that the operator $B_{h}$ is treated using an explicit two-stage RK scheme and the operator $A_{h}$ using a diagonally implicit two-stage RK scheme. By using equation (2.8a) in (2.8b) and equations (2.8a)-(2.8b) in (2.8c), we obtain the following alternative form of the system (2.8):

$$
\begin{aligned}
v_{h}^{n} & =u_{h}^{n}-\gamma \tau A_{h} v_{h}^{n}+\gamma \tau f_{h}^{n}, \\
w_{h}^{n} & =v_{h}^{n}-\tau B_{h} v_{h}^{n}-(1-3 \gamma) \tau A_{h} v_{h}^{n}-\gamma \tau A_{h} w_{h}^{n}+(1-2 \gamma) \tau f_{h}^{n}, \\
u_{h}^{n+1} & =\frac{1}{2}\left(v_{h}^{n}+w_{h}^{n}\right)-\frac{1}{2} \tau B_{h} w_{h}^{n}-\frac{1}{2} \gamma \tau A_{h} v_{h}^{n}-\frac{1}{2}(1-\gamma) \tau A_{h} w_{h}^{n}+\tau\left(f_{h}^{n+\frac{1}{2}}-\frac{1}{2} f_{h}^{n}\right) .
\end{aligned}
$$

\subsection{Truncation error in time}

The goal of this section is to identify the truncation error in time. Recalling the operators $B: V \ni v \mapsto \beta \cdot \nabla v \in$ $L$ and $A: V \ni v \mapsto-\mu \Delta v \in L$, we introduce, for all $0 \leq n \leq N-1$, the auxiliary functions $v^{n}, w^{n} \in H_{0}^{1}(\Omega)$ 
such that (compare with $(2.8 \mathrm{a})-(2.8 \mathrm{~b}))$

$$
\begin{aligned}
v^{n}+\gamma \tau A v^{n} & =u^{n}+\gamma \tau f^{n}, \\
w^{n}+\gamma \tau A w^{n} & =u^{n}-\tau B v^{n}-(1-2 \gamma) \tau A v^{n}+(1-\gamma) \tau f^{n},
\end{aligned}
$$

or, equivalently, subtracting (2.10a) from (2.10b) (compare with (2.9b))

$$
w^{n}+\gamma \tau A w^{n}=v^{n}-\tau B v^{n}-(1-3 \gamma) \tau A v^{n}+(1-2 \gamma) \tau f^{n} .
$$

Moreover, owing to elliptic regularity, $v^{n}, w^{n} \in V$.

Definition 2.2 (truncation error). The truncation error $\Psi^{n} \in L$ at the discrete time $n \tau$ is defined as

$$
\Psi^{n}:=\tau^{-1}\left(u^{n+1}-u^{n}\right)+\frac{1}{2}(A+B)\left(v^{n}+w^{n}\right)-f^{n+1 / 2} .
$$

It is straightforward to verify that (compare with (2.8c) and (2.9c))

$$
\begin{aligned}
u^{n+1} & =u^{n}-\frac{1}{2} \tau B\left(v^{n}+w^{n}\right)-\frac{1}{2} \tau A\left(v^{n}+w^{n}\right)+\tau f^{n+1 / 2}+\tau \Psi^{n} \\
& =\frac{1}{2}\left(v^{n}+w^{n}\right)-\frac{1}{2} \tau B w^{n}-\frac{1}{2} \gamma \tau A v^{n}-\frac{1}{2}(1-\gamma) \tau A w^{n}+\tau\left(f^{n+1 / 2}-\frac{1}{2} f^{n}\right)+\tau \Psi^{n} .
\end{aligned}
$$

\subsection{Error equation}

To formulate the error equation, we define

$$
\begin{array}{lll}
\xi_{h}^{n}=u_{h}^{n}-\pi_{h} u^{n}, & \theta_{h}^{n}=v_{h}^{n}-\pi_{h} v^{n}, & \zeta_{h}^{n}=w_{h}^{n}-\pi_{h} w^{n}, \\
\xi_{\pi}^{n}=u^{n}-\pi_{h} u^{n}, & \theta_{\pi}^{n}=v^{n}-\pi_{h} v^{n}, & \zeta_{\pi}^{n}=w^{n}-\pi_{h} w^{n} .
\end{array}
$$

Hence, the approximation error can be written as $u^{n}-u_{h}^{n}=-\xi_{h}^{n}+\xi_{\pi}^{n}$ and similarly for $v^{n}-v_{h}^{n}$ and $w^{n}-w_{h}^{n}$. The functions $\xi_{\pi}^{n}, \theta_{\pi}^{n}$, and $\zeta_{\pi}^{n}$ are used to measure the space approximation errors.

Lemma 2.3 (error equation). There holds

$$
\begin{aligned}
\theta_{h}^{n} & =\xi_{h}^{n}-\gamma \tau A_{h} \theta_{h}^{n}+\tau \alpha_{h}^{n}, \\
\zeta_{h}^{n} & =\theta_{h}^{n}-\tau B_{h} \theta_{h}^{n}-(1-3 \gamma) \tau A_{h} \theta_{h}^{n}-\gamma \tau A_{h} \zeta_{h}^{n}+\tau \beta_{h}^{n}, \\
\xi_{h}^{n+1} & =\frac{1}{2}\left(\theta_{h}^{n}+\zeta_{h}^{n}\right)-\frac{1}{2} \tau B_{h} \zeta_{h}^{n}-\frac{1}{2} \gamma \tau A_{h} \theta_{h}^{n}-\frac{1}{2}(1-\gamma) \tau A_{h} \zeta_{h}^{n}+\tau \delta_{h}^{n}-\tau \Psi_{h}^{n},
\end{aligned}
$$

where $\Psi_{h}^{n}:=\pi_{h} \Psi^{n}$ and

$$
\alpha_{h}^{n}=\gamma A_{h} \theta_{\pi}^{n}, \quad \beta_{h}^{n}=B_{h} \theta_{\pi}^{n}+(1-3 \gamma) A_{h} \theta_{\pi}^{n}+\gamma A_{h} \zeta_{\pi}^{n}, \quad \delta_{h}^{n}=\frac{1}{2} B_{h} \zeta_{\pi}^{n}+\frac{1}{2} \gamma A_{h} \theta_{\pi}^{n}+\frac{1}{2}(1-\gamma) A_{h} \zeta_{\pi}^{n} .
$$

Proof. Apply the projector $\pi_{h}$ to (2.10a), (2.11), and (2.13), use consistency, and subtract the resulting equations from (2.9).

\subsection{Stability and boundedness of the discrete operators $A_{h}$ and $B_{h}$}

We define the following seminorm and norm on $V(h)$,

$$
\begin{aligned}
|z|_{S}^{2} & :=\sum_{F \in \mathcal{F}_{h}^{\text {int }}} S_{\text {cip }} h_{F}^{2}\left\|\left.\nu_{F} \cdot \beta\right|^{1 / 2} \nu_{F} \cdot \llbracket \nabla z \rrbracket\right\|_{L, F}^{2}, \\
\|z\|_{A}^{2} & :=\mu\|\nabla z\|_{L^{d}}^{2}+\mu h^{-1}\|z\|_{L, \partial \Omega}^{2} .
\end{aligned}
$$


It is well-known that provided $S_{\mathrm{bc}}$ is sufficiently large, there is $c_{a}>0$ such that for all $v_{h} \in V_{h}$,

$$
\left(A_{h} v_{h}, v_{h}\right)_{L} \geq c_{a}\left\|v_{h}\right\|_{A}^{2} .
$$

To allow for a more compact notation, we also consider the norm $\left\|v_{h}\right\|_{a}:=\left(A_{h} v_{h}, v_{h}\right)_{L}^{1 / 2}$ for all $v_{h} \in V_{h}$. Furthermore, integration by parts readily yields

$$
\left(B_{h} v_{h}, v_{h}\right)_{L}=\left|v_{h}\right|_{S}^{2}
$$

We now examine briefly some important boundedness properties of the discrete operators $A_{h}$ and $B_{h}$. In addition to the $|\cdot|_{S}$-seminorm and the $\|\cdot\|_{A}$-norm defined above, we consider the following norms on $V(h)$,

$$
\begin{aligned}
\|z\|_{B *} & :=|z|_{S}+\sigma^{1 / 2} h^{-1 / 2}\|z\|_{L}, \\
\|z\|_{A *} & :=\|z\|_{A}+\mu^{1 / 2} h^{1 / 2}\|\nu \cdot \nabla z\|_{L, \partial \Omega} .
\end{aligned}
$$

These norms will be used to measure the space approximation errors. The following properties of $B_{h}$ are established in $[5,7,8]$. Note that property $(2.22)$ is valid only for piecewise affine functions.

Lemma 2.4 (boundedness of $B_{h}$ ). For all $z \in V(h)$,

$$
\left\|B_{h} z\right\|_{L} \leq \sigma\|\nabla z\|_{L^{d}}+C_{S} \sigma^{1 / 2} h^{-1 / 2}|z|_{S},
$$

for all $\left(z, v_{h}\right) \in V(h) \times V_{h}$,

$$
\left|\left(B_{h}\left(z-\pi_{h} z\right), v_{h}\right)_{L}\right| \lesssim\left\|z-\pi_{h} z\right\|_{B *}\left(\left|v_{h}\right|_{S}+\sigma_{1}^{1 / 2}\left\|v_{h}\right\|_{L}\right),
$$

and for all $\left(v_{h}, w_{h}\right) \in V_{h} \times V_{h}$,

$$
\left|\left(B_{h} v_{h}, w_{h}-\pi_{h}^{0} w_{h}\right)_{L}\right| \leq C_{B} \sigma^{1 / 2} h^{-1 / 2}\left(\left|v_{h}\right|_{S}+\sigma_{1}^{1 / 2}\left\|v_{h}\right\|_{L}\right)\left\|w_{h}-\pi_{h}^{0} w_{h}\right\|_{L},
$$

where $\pi_{h}^{0}$ denotes the L-orthogonal projection onto piecewise constant functions.

Using discrete trace and inverse inequalities, together with (2.20) yields for all $v_{h} \in V_{h}$,

$$
\left|v_{h}\right|_{S} \lesssim \sigma^{1 / 2} h^{-1 / 2}\left\|v_{h}\right\|_{L}, \quad\left\|B_{h} v_{h}\right\|_{L} \lesssim \sigma h^{-1}\left\|v_{h}\right\|_{L},
$$

while using (2.21) and the previous bound on $\left|v_{h}\right|_{S}$ yields for all $z \in V(h)$,

$$
\tau\left\|B_{h}\left(z-\pi_{h} z\right)\right\|_{L} \lesssim \tau^{1 / 2} \mathrm{Co}^{1 / 2}\left\|z-\pi_{h} z\right\|_{B *} .
$$

The following properties of $A_{h}$ are established using fairly standard arguments, in particular discrete trace and inverse inequalities and the uniform equivalence of the $\|\cdot\|_{A^{-}}$and $\|\cdot\|_{A^{*}}$-norms on $V_{h}$.

Lemma 2.5 (boundedness of $\left.A_{h}\right)$. For all $\left(z, w_{h}\right) \in V(h) \times V_{h}$,

$$
\left|\left(A_{h} z, w_{h}\right)_{L}\right| \lesssim\|z\|_{A *}\left\|w_{h}\right\|_{A} \quad \text { so that } \quad\left\|A_{h} z\right\|_{L} \lesssim \mu^{1 / 2} h^{-1}\|z\|_{A *} .
$$

Additionally, for all $\left(z_{h}, w_{h}\right) \in V_{h} \times V_{h}$,

$$
\left|\left(A_{h} z_{h}, w_{h}\right)_{L}\right| \lesssim\left\|z_{h}\right\|_{A}\left\|w_{h}\right\|_{A} \quad \text { so that } \quad\left\|A_{h} z_{h}\right\|_{L} \lesssim \mu^{1 / 2} h^{-1}\left\|z_{h}\right\|_{A} .
$$

\section{TRunCation AND SPACE APPROXimation ERRORS}

The goal of this section is to establish bounds on the truncation error $\Psi^{n}$ defined by (2.12) and on the space approximation errors associated with the functions $\theta_{\pi}^{n}$ and $\zeta_{\pi}^{n}$ defined by (2.14b). To this end, we first derive bounds on the auxiliary functions at intermediate stages, namely the functions $v^{n}$ and $w^{n}$ defined by (2.10). Recall that owing to elliptic regularity, these functions are in $V=H^{2}(\Omega) \cap H_{0}^{1}(\Omega)$. 


\subsection{Bounds on the auxiliary functions at intermediate stages}

Bounding Sobolev norms of the functions $v^{n}$ and $w^{n}$ hinges on the stability properties of the operator $(I+\gamma \tau A)^{-1}$ (where $I$ is the identity in $\left.V\right)$.

Lemma 3.1 (stability of $(I+\gamma \tau A))$. Let $\mathfrak{v} \in L$ and let $\mathfrak{u} \in V$ be such that

$$
(I+\gamma \tau A) \mathfrak{u}=\mathfrak{v} .
$$

Then,

$$
\|\mathfrak{u}\|_{L} \lesssim\|\mathfrak{v}\|_{L}, \quad(\mu \tau)^{1 / 2}\|\nabla \mathfrak{u}\|_{L^{d}} \lesssim\|\mathfrak{v}\|_{L}
$$

If, additionally $\mathfrak{v} \in H_{0}^{1}(\Omega)$,

$$
\|\nabla \mathfrak{u}\|_{L^{d}} \lesssim\|\nabla \mathfrak{v}\|_{L^{d}}, \quad(\mu \tau)^{1 / 2}\|\Delta \mathfrak{u}\|_{L} \lesssim\|\nabla \mathfrak{v}\|_{L^{d}}
$$

If, additionally $\mathfrak{v} \in V$,

$$
\|\Delta \mathfrak{u}\|_{L} \lesssim\|\Delta \mathfrak{v}\|_{L}, \quad(\mu \tau)^{1 / 2}\|\nabla \Delta \mathfrak{u}\|_{L^{d}} \lesssim\|\Delta \mathfrak{v}\|_{L} .
$$

Proof. Take the $L$-scalar product of (3.1) with $\mathfrak{u}$ and integrate by parts to infer (3.2), apply the same procedure to (3.1) with $\Delta \mathfrak{u}$ observing that $\left.\Delta \mathfrak{u}\right|_{\partial \Omega}=0$ owing to (3.1) to infer (3.3), and take the Laplacian of (3.1) and apply the same procedure with $\Delta \mathfrak{u}$ to infer (3.4).

As a first application, we derive bounds on $\left(v^{n}-u^{n}\right)$ and on $v^{n}$.

Lemma 3.2 (bounds on $\left(v^{n}-u^{n}\right)$ and $\left.v^{n}\right)$. For $s \in\{1,2\}$, set $K_{s}^{n}:=\left|f^{n}\right|_{H^{s}}+\mu\left|u^{n}\right|_{H^{s+2}}$. Then,

$$
\left\|\nabla\left(v^{n}-u^{n}\right)\right\|_{L^{d}} \lesssim \tau K_{1}^{n}, \quad\left\|\Delta\left(v^{n}-u^{n}\right)\right\|_{L} \lesssim \tau K_{2}^{n}, \quad(\mu \tau)^{1 / 2}\left\|\nabla \Delta\left(v^{n}-u^{n}\right)\right\|_{L} \lesssim \tau K_{2}^{n},
$$

and letting $\tilde{K}_{s}^{n}=\left|u^{n}\right|_{H^{s}}+\tau K_{s}^{n}$,

$$
\left\|\nabla v^{n}\right\|_{L^{d}} \lesssim \tilde{K}_{1}^{n}, \quad\left|v^{n}\right|_{H^{2}} \lesssim \tilde{K}_{2}^{n} .
$$

Proof. Take $\mathfrak{u}:=v^{n}-u^{n}$ so that $\mathfrak{v}=\gamma \tau\left(f^{n}-A u^{n}\right)$ owing to (2.10a). Since $\mathfrak{v} \in V$ (recall that $f^{n}$ and $A u^{n}$ vanish on $\partial \Omega$ ), the bound on $\left\|\nabla\left(v^{n}-u^{n}\right)\right\|_{L^{d}}$ results from (3.3) and the two other bounds on $\left(v^{n}-u^{n}\right)$ from (3.4). Finally, the bounds (3.6) on $v^{n}$ result from (3.5), the triangle inequality, and elliptic regularity.

As a second application, we derive bounds on $\left(w^{n}-u^{n}\right)$ and on $w^{n}$.

Lemma 3.3 (bounds on $\left(w^{n}-u^{n}\right)$ and $\left.w^{n}\right)$. Let $K_{w-u}^{n}:=K_{1}^{n}+\sigma \tilde{K}_{2}^{n}+\sigma_{1} \tilde{K}_{1}^{n}$. Then,

$$
\left\|\nabla\left(w^{n}-u^{n}\right)\right\|_{L^{d}} \lesssim \tau K_{w-u}^{n}, \quad(\mu \tau)^{1 / 2}\left\|\Delta\left(w^{n}-u^{n}\right)\right\|_{L} \lesssim \tau K_{w-u}^{n},
$$

and

$$
(\mu \tau)^{1 / 2}\left|w^{n}\right|_{H^{2}} \lesssim(\mu \tau)^{1 / 2}\left|u^{n}\right|_{H^{2}}+\tau K_{w-u}^{n} .
$$

Proof. We first deduce from (2.10) that

$$
(I+\gamma \tau A)\left(w^{n}-u^{n}\right)=\gamma \tau\left(f^{n}-A u^{n}\right)+\gamma^{-1}(1-2 \gamma)\left(v^{n}-u^{n}\right)-\tau B v^{n} .
$$

As a result, we can apply Lemma 3.1 with $\mathfrak{u}:=w^{n}-u^{n}$ and $\mathfrak{v}$ equal to the right-hand side of (3.9). We observe that $\mathfrak{v} \in H_{0}^{1}(\Omega)$ and that $\|\nabla \mathfrak{v}\|_{L^{d}} \lesssim \tau K_{w-u}^{n}$ since, in particular, $\left\|\nabla\left(B v^{n}\right)\right\|_{L^{d}} \leq \sigma\left|v^{n}\right|_{H^{2}}+\sigma_{1}\left\|\nabla v^{n}\right\|_{L^{d}} \lesssim$ $\sigma \tilde{K}_{2}^{n}+\sigma_{1} \tilde{K}_{1}^{n}$ where we have used (3.6) to bound $v^{n}$. Hence, the bounds (3.7) on $\left(w^{n}-u^{n}\right)$ result from (3.3). Finally, the bound (3.8) on $w^{n}$ results from (3.7), the triangle inequality, and elliptic regularity. 


\subsection{Bound on the truncation error}

In this section, we derive two bounds on the truncation error. To this end, it is useful to consider the following equivalent expression for $\Psi^{n}$ (the proof, which amounts to a direct verification, is skipped for brevity).

Lemma 3.4 (equivalent expression for $\Psi^{n}$ ). Let $x^{n} \in V$ be defined such that

$$
x^{n}:=\frac{1}{2}\left(v^{n}+w^{n}\right)-u^{n}-\frac{1}{2} \tau \partial_{t} u^{n} .
$$

Then, letting $\tilde{\Psi}^{n}:=\tau^{-1}\left(u^{n+1}-u^{n}-\tau \partial_{t} u^{n}-\frac{1}{2} \tau^{2} \partial_{t t} u^{n}\right)+\left(f^{n}+\frac{1}{2} \tau \partial_{t} f^{n}-f^{n+1 / 2}\right)$, there holds

$$
\Psi^{n}=\tilde{\Psi}^{n}+B x^{n}+A x^{n} .
$$

We observe that it is necessary to bound spatial derivatives of $x^{n}$ in order to control the terms $B x^{n}$ and $A x^{n}$. Here, the bounds on $\left(v^{n}-u^{n}\right)$ derived in Lemma 3.2 are instrumental.

Lemma 3.5 (bounds on $\left.x^{n}\right)$. Let $C_{x}^{n}:=\mu^{1 / 2} K_{2}^{n}+\tau^{1 / 2}\left(\sigma K_{2}^{n}+\sigma_{1} K_{1}^{n}+\mu\left|\partial_{t} u^{n}\right|_{H^{3}}\right)$. Then,

$$
\begin{aligned}
\left\|B x^{n}\right\|_{L} & \lesssim \sigma C_{x}^{n} \tau^{3 / 2}, \\
\left\|A x^{n}\right\|_{L} & \lesssim \mu^{1 / 2} C_{x}^{n} \tau, \\
\left\|x^{n}\right\|_{A *} & \lesssim \bar{\mu}^{1 / 2} C_{x}^{n} \tau^{3 / 2} .
\end{aligned}
$$

Proof. A direct calculation shows that

$$
y^{n}:=(I+\gamma \tau A) x^{n}=-\frac{1}{2} \tau B\left(v^{n}-u^{n}\right)-\frac{1}{2}(1-2 \gamma) \tau A\left(v^{n}-u^{n}\right)-\frac{1}{2} \gamma \tau^{2} A \partial_{t} u^{n} .
$$

Applying Lemma 3.1 with $\mathfrak{u}=x^{n}$ and $\mathfrak{v}=y^{n}$ and observing that $y^{n} \in H_{0}^{1}(\Omega)$ (for the first term, $\nu \cdot \beta$ as well as $\left(v^{n}-u^{n}\right)$ vanish on $\partial \Omega$; for the second term, $A v^{n}$ vanishes on $\partial \Omega$ owing to (2.10a) and $A u^{n}$ by Proposition 2.1; for the third term, $A u(t)$ vanishes on $\partial \Omega$ at all times by Proposition 2.1 and, hence, so does its time-derivative), we infer using (3.3) that $\left\|\nabla x^{n}\right\|_{L^{d}} \lesssim\left\|\nabla y^{n}\right\|_{L^{d}}$ and $(\mu \tau)^{1 / 2}\left\|\Delta x^{n}\right\|_{L} \lesssim\left\|\nabla y^{n}\right\|_{L^{d}}$. Using the bounds (3.5) on $\left(v^{n}-u^{n}\right)$ yields $\left\|\nabla y^{n}\right\|_{L^{d}} \lesssim C_{x}^{n} \tau^{3 / 2}$, whence (3.12a) and (3.12b). Finally, a continuous scaled trace inequality together with elliptic regularity yield

$$
\left\|x^{n}\right\|_{A *} \lesssim \mu^{1 / 2}\left(\left\|\nabla x^{n}\right\|_{L^{d}}+h\left|x^{n}\right|_{H^{2}}\right) \lesssim \mu^{1 / 2}\left(\left\|\nabla x^{n}\right\|_{L^{d}}+h\left\|\Delta x^{n}\right\|_{L}\right) .
$$

Using the reverse-parabolic CFL inequality (2.3) and the above bounds on $\left\|\nabla x^{n}\right\|_{L^{d}}$ and $\left\|\Delta x^{n}\right\|_{L}$, we infer

$$
\left\|x^{n}\right\|_{A *} \lesssim \bar{\mu}^{1 / 2}\left(\left\|\nabla x^{n}\right\|_{L^{d}}+(\mu \tau)^{1 / 2}\left\|\Delta x^{n}\right\|_{L}\right) \lesssim \bar{\mu}^{1 / 2}\left\|\nabla y^{n}\right\|_{L^{d}}
$$

whence (3.12c) results from the bound on $\left\|\nabla y^{n}\right\|_{L^{d}}$.

We can now state the main result of this section, providing two ways to bound the truncation error. The first bound (3.15a) is simpler, but is only first-order in time; the second bound (3.15b) is of higher-order, namely $3 / 2$, but estimates the diffusive contribution of $x^{n}$ differently. Both bounds will be used in what follows.

Lemma 3.6. Let

$$
\begin{aligned}
& C_{\Psi}^{n}:=\left(t_{*} \tau\right)^{1 / 2} C_{u, f}^{n}+t_{*}^{1 / 2} \sigma C_{x}^{n}+\bar{\mu}^{1 / 2} C_{x}^{n}, \\
& \tilde{C}_{\Psi}^{n}:=\tau C_{u, f}^{n}+\tau^{1 / 2} \sigma C_{x}^{n}+\mu^{1 / 2} C_{x}^{n},
\end{aligned}
$$

where $C_{x}^{n}$ is defined in Lemma 3.5 and $C_{u, f}^{n}:=\|u\|_{C^{3}\left(I_{n} ; L\right)}+\|f\|_{C^{2}\left(I_{n} ; L\right)}$. Then,

$$
\begin{aligned}
&\left\|\Psi^{n}\right\|_{L} \leq\left\|\tilde{\Psi}^{n}\right\|_{L}+\left\|B x^{n}\right\|_{L}+\left\|A x^{n}\right\|_{L} \lesssim \tilde{C}_{\Psi}^{n} \tau \\
&\left\|\tilde{\Psi}^{n}\right\|_{L}+\left\|B x^{n}\right\|_{L}+t_{*}^{-1 / 2}\left\|x^{n}\right\|_{A *} \lesssim t_{*}^{-1 / 2} C_{\Psi}^{n} \tau^{3 / 2} .
\end{aligned}
$$


Proof. Using the definition (3.11) and the triangle inequality leads to

$$
\left\|\Psi^{n}\right\|_{L} \leq\left\|\tilde{\Psi}^{n}\right\|_{L}+\left\|B x^{n}\right\|_{L}+\left\|A x^{n}\right\|_{L},
$$

whence (3.15a) results from (3.12a), (3.12b), and the obvious bound $\left\|\tilde{\Psi}^{n}\right\|_{L} \leq C_{u, f}^{n} \tau^{2}$. Furthermore, the second bound (3.15b) results from (3.12a), (3.12c), and the same bound on $\left\|\tilde{\Psi}^{n}\right\|_{L}$.

Remark 3.7 (convergence order in time). Although the two-stage IMEX RK scheme is formally second-order, as reflected by the bound on $\left\|\tilde{\Psi}^{n}\right\|_{L}$ based on Taylor polynomial expansions on $u$ and $f$, the bounds on the truncation error derived in Lemma 3.6 are not second-order. In fact, although $\left\|x^{n}\right\|_{L}$ is second-order in time (this results from (3.13) so that $\left\|x^{n}\right\|_{L} \leq\left\|y^{n}\right\|_{L}$ and the fact that $\left.\left\|y^{n}\right\|_{L} \lesssim \tau^{2}\left(\sigma K_{1}+\mu K_{2}+\mu\left|\partial_{t} u^{n}\right|_{H^{2}}\right)\right)$, the first- and second-order derivatives of $x^{n}$ are not second-order in time, as reflected by the bounds derived in Lemma 3.5 on $\left\|B x^{n}\right\|_{L}$ and $\left\|A x^{n}\right\|_{L}$. The difficulty in deriving higher-order bounds on $\left\|B x^{n}\right\|_{L}$ and $\left\|A x^{n}\right\|_{L}$ stems from boundary conditions. To establish the present bounds, we have, in particular, made use of $\left.A u^{n}\right|_{\partial \Omega}=0$ and $\left.B u^{n}\right|_{\partial \Omega}=0$ owing to Proposition 2.1. Under the more restrictive assumption $\left.A B u^{n}\right|_{\partial \Omega}=0$ (which holds true, e.g., if the normal derivative of $\beta$ and the Laplacian of the normal component of $\beta$ vanish on $\partial \Omega$ ), it is possible to gain a factor $\tau^{1 / 2}$ in the bounds on $\left\|B x^{n}\right\|_{L}$ and $\left\|A x^{n}\right\|_{L}$. This results from the fact that the function $y^{n}$ defined by (3.13) is such that $(I+\gamma \tau A) y^{n}=\tau^{2}\left(z_{1}^{n}+z_{2}^{n}\right)$ with $z_{1}^{n}=-\frac{1}{2} \gamma B\left(f^{n}-A u^{n}\right)-\frac{1}{2}(1-2 \gamma) \gamma A\left(f^{n}-\right.$ $\left.A u^{n}\right)-\frac{1}{2} \gamma A \partial_{t} u^{n} \in H_{0}^{1}(\Omega)$ (since $\left.\left.A B u^{n}\right|_{\partial \Omega}=0\right)$ and $z_{2}^{n}=-\frac{1}{2} \gamma(A B-B A)\left(v^{n}-u^{n}\right)-\frac{1}{2} \gamma^{2} \tau A^{2} \partial_{t} u^{n} \in L$ so that $\left\|\nabla y^{n}\right\|_{L^{d}} \lesssim \tau^{2}$ (details are skipped for brevity). An alternative assumption leading to the same conclusion is to use periodic boundary conditions. Finally, we stress that the present bounds are, however, sufficient to equilibrate the space and time errors in our error estimates in the context of the CFL restriction on the time step.

\subsection{Bounds on the space approximation errors}

The goal of this section is to bound the $\|\cdot\|_{A *^{-}}$and $\|\cdot\|_{B *^{-}}$norms of $\theta_{\pi}^{n}$ and $\zeta_{\pi}^{n}$. We first observe that standard approximation properties in finite element spaces yield for all $z \in H^{2}(\Omega)$,

$$
\left\|z-\pi_{h} z\right\|_{B *} \lesssim \sigma^{1 / 2} h^{3 / 2}|z|_{H^{2}}, \quad\left\|z-\pi_{h} z\right\|_{A *} \lesssim \mu^{1 / 2} h|z|_{H^{2}} .
$$

Lemma 3.8 (bound on $\theta_{\pi}^{n}$ and $\zeta_{\pi}^{n}$ ). There holds

$$
\begin{aligned}
\left\|\theta_{\pi}^{n}\right\|_{B *}+\left\|\theta_{\pi}^{n}\right\|_{A *} & \lesssim\left(\sigma^{1 / 2} h^{3 / 2}+\mu^{1 / 2} h\right) \tilde{K}_{2}^{n} \\
\left\|\zeta_{\pi}^{n}\right\|_{B *}+\left\|\zeta_{\pi}^{n}\right\|_{A *} & \lesssim\left(\sigma^{1 / 2} h^{3 / 2}+\mu^{1 / 2} h\right) \tilde{K}_{2}^{n}+\tau^{1 / 2} h K_{w-u}^{n}
\end{aligned}
$$

Proof. The bound (3.17a) readily results from (3.16) and the bound (3.6) on $\left|v^{n}\right|_{H^{2}}$. To bound $\left\|\zeta_{\pi}^{n}\right\|_{A *}$, we use again (3.16) together with (3.8) yielding $\left\|\zeta_{\pi}^{n}\right\|_{A *} \lesssim \mu^{1 / 2} h\left|u^{n}\right|_{H^{2}}+\tau^{1 / 2} h K_{w-u}^{n}$. To bound $\left\|\zeta_{\pi}^{n}\right\|_{B *}$, we first observe that for a function $z \in V$,

$$
\left\|z-\pi_{h} z\right\|_{B *} \lesssim \sigma^{1 / 2} h^{1 / 2}\|\nabla z\|_{L^{d}} .
$$

This assertion is clear for the $\|\cdot\|_{L}$-norm contribution, while using a discrete trace inequality and the $H^{1}$-stability of $\pi_{h}$ yields

$$
\left|z-\pi_{h} z\right|_{S}=\left|\pi_{h} z\right|_{S} \lesssim \sigma^{1 / 2} h^{1 / 2}\left\|\nabla \pi_{h} z\right\|_{L^{d}} \lesssim \sigma^{1 / 2} h^{1 / 2}\|\nabla z\|_{L^{d}} .
$$

As a result, starting from the triangle inequality

$$
\left\|\zeta_{\pi}^{n}\right\|_{B *} \leq\left\|u^{n}-\pi_{h} u^{n}\right\|_{B *}+\left\|\left(w^{n}-u^{n}\right)-\pi_{h}\left(w^{n}-u^{n}\right)\right\|_{B *},
$$

and using the approximation property (3.16) for the first term, together with (3.18) and (3.7) to bound $\| \nabla\left(w^{n}-\right.$ $\left.u^{n}\right) \|_{L^{d}}$, we infer

$$
\left\|\zeta_{\pi}^{n}\right\|_{B *} \lesssim \sigma^{1 / 2} h^{3 / 2}\left|u^{n}\right|_{H^{2}}+\sigma^{1 / 2} h^{1 / 2} \tau K_{w-u}^{n} \leq \sigma^{1 / 2} h^{3 / 2}\left|u^{n}\right|_{H^{2}}+\tau^{1 / 2} h K_{w-u}^{n},
$$

where we have used Co $\leq 1$. The conclusion is straightforward since $\left|u^{n}\right|_{H^{2}} \leq \tilde{K}_{2}^{n}$. 


\section{Stability AND CONVERGEnCE ANALYSiS}

This section is devoted to the stability and convergence analysis of the IMEX RK scheme (2.9). Firstly, we derive a basic energy estimate valid in all flow regimes (Thm. 4.4). On the right-hand side of this estimate appears an anti-dissipative term together with the time and space discretization errors. Then, we bound the anti-dissipative term depending on the flow regime, yielding our main convergence results (Thms. 4.8 and 4.16 together with Props. 4.9 and 4.17).

\subsection{Basic energy identity}

We begin the analysis with a basic energy identity valid in all flow regimes.

Lemma 4.1 (basic energy identity). Assume $\gamma \in\left(0, \frac{1}{2}\right)$. There holds

$$
\begin{aligned}
& \frac{1}{2}\left\|\xi_{h}^{n+1}\right\|_{L}^{2}-\frac{1}{2}\left\|\xi_{h}^{n}\right\|_{L}^{2}+\frac{1}{2}\left\|\theta_{h}^{n}-\xi_{h}^{n}\right\|_{L}^{2}+\frac{1}{2} \tau\left|\theta_{h}^{n}\right|_{S}^{2}+\frac{1}{2} \tau\left|\zeta_{h}^{n}\right|_{S}^{2}+\left(\frac{1}{2}-\gamma\right) \tau\left\|\theta_{h}^{n}\right\|_{a}^{2}+\left(\frac{1}{2}-\gamma\right) \tau\left\|\zeta_{h}^{n}\right\|_{a}^{2} \\
& +\frac{1}{2} \gamma \tau\left\|\zeta_{h}^{n}+\theta_{h}^{n}\right\|_{a}^{2}=\frac{1}{2}\left\|\xi_{h}^{n+1}-\zeta_{h}^{n}\right\|_{L}^{2}+\tau\left(\alpha_{h}^{n}+\frac{1}{2} \beta_{h}^{n}, \theta_{h}^{n}\right)_{L}+\tau\left(\delta_{h}^{n}, \zeta_{h}^{n}\right)_{L}-\tau\left(\Psi_{h}^{n}, \zeta_{h}^{n}\right)_{L} .
\end{aligned}
$$

Remark 4.2 (pure advection, role of diffusion). Setting the diffusion coefficient to zero, the energy identity (4.1) reduces to the one derived in [8] for explicit RK2 schemes in the purely advective case. Moreover, in the presence of diffusion, all the additional terms involving the $\|\cdot\|_{a}$-norm are dissipative for $\gamma \in\left(0, \frac{1}{2}\right)$.

Proof. We multiply equation (2.15a) by $\theta_{h}^{n}$ to obtain using the discrete stability (2.17) of $A_{h}$,

$$
\frac{1}{2}\left\|\theta_{h}^{n}\right\|_{L}^{2}+\frac{1}{2}\left\|\theta_{h}^{n}-\xi_{h}^{n}\right\|_{L}^{2}=\frac{1}{2}\left\|\xi_{h}^{n}\right\|_{L}^{2}+\left(\theta_{h}^{n}-\xi_{h}^{n}, \theta_{h}^{n}\right)_{L}=\frac{1}{2}\left\|\xi_{h}^{n}\right\|_{L}^{2}-\gamma \tau\left\|\theta_{h}^{n}\right\|_{a}^{2}+\tau\left(\alpha_{h}^{n}, \theta_{h}^{n}\right)_{L} .
$$

Then, we multiply equation $(2.15 \mathrm{~b})$ by $\frac{1}{2} \theta_{h}^{n}$ and equation $(2.15 \mathrm{c})$ by $\zeta_{h}^{n}$ to obtain

$$
\frac{1}{2}\left(\zeta_{h}^{n}, \theta_{h}^{n}\right)_{L}=\frac{1}{2}\left\|\theta_{h}^{n}\right\|_{L}^{2}-\frac{1}{2} \tau\left(B_{h} \theta_{h}^{n}, \theta_{h}^{n}\right)_{L}-\frac{1}{2}(1-3 \gamma) \tau\left\|\theta_{h}^{n}\right\|_{a}^{2}-\frac{1}{2} \gamma \tau\left(A_{h} \zeta_{h}^{n}, \theta_{h}^{n}\right)_{L}+\frac{1}{2} \tau\left(\beta_{h}^{n}, \theta_{h}^{n}\right)_{L}
$$

and

$$
\left(\xi_{h}^{n+1}, \zeta_{h}^{n}\right)_{L}=\frac{1}{2}\left(\theta_{h}^{n}+\zeta_{h}^{n}, \zeta_{h}^{n}\right)_{L}-\frac{1}{2} \tau\left(B_{h} \zeta_{h}^{n}, \zeta_{h}^{n}\right)_{L}-\frac{1}{2} \gamma \tau\left(A_{h} \theta_{h}^{n}, \zeta_{h}^{n}\right)_{L}-\frac{1}{2}(1-\gamma) \tau\left\|\zeta_{h}^{n}\right\|_{a}^{2}+\tau\left(\delta_{h}^{n}-\Psi_{h}^{n}, \zeta_{h}^{n}\right)_{L} .
$$

Summing (4.2) and (4.3) we deduce

$$
\begin{aligned}
\frac{1}{2}\left(\zeta_{h}^{n}, \theta_{h}^{n}\right)_{L}= & -\frac{1}{2}\left\|\theta_{h}^{n}-\xi_{h}^{n}\right\|_{L}^{2}+\frac{1}{2}\left\|\xi_{h}^{n}\right\|_{L}^{2}-\frac{1}{2} \tau\left(B_{h} \theta_{h}^{n}, \theta_{h}^{n}\right)_{L}-\frac{1}{2}(1-\gamma) \tau\left\|\theta_{h}^{n}\right\|_{a}^{2}-\frac{1}{2} \gamma \tau\left(A_{h} \zeta_{h}^{n}, \theta_{h}^{n}\right)_{L} \\
& +\tau\left(\alpha_{h}^{n}+\frac{1}{2} \beta_{h}^{n}, \theta_{h}^{n}\right)_{L} .
\end{aligned}
$$

Using now the identity $\left(\xi_{h}^{n+1}, \zeta_{h}^{n}\right)_{L}=\frac{1}{2}\left\|\xi_{h}^{n+1}\right\|_{L}^{2}-\frac{1}{2}\left\|\xi_{h}^{n+1}-\zeta_{h}^{n}\right\|_{L}^{2}+\frac{1}{2}\left\|\zeta_{h}^{n}\right\|_{L}^{2}$ together with (4.4) and (4.5), we infer

$$
\begin{aligned}
& \frac{1}{2}\left\|\xi_{h}^{n+1}\right\|_{L}^{2}-\frac{1}{2}\left\|\xi_{h}^{n+1}-\zeta_{h}^{n}\right\|_{L}^{2}+\frac{1}{2}\left\|\zeta_{h}^{n}\right\|_{L}^{2}=\frac{1}{2}\left\|\zeta_{h}^{n}\right\|_{L}^{2}-\frac{1}{2}\left\|\theta_{h}^{n}-\xi_{h}^{n}\right\|_{L}^{2}+\frac{1}{2}\left\|\xi_{h}^{n}\right\|_{L}^{2}-\frac{1}{2} \tau\left(B_{h} \theta_{h}^{n}, \theta_{h}^{n}\right)_{L}-\frac{1}{2} \tau\left(B_{h} \zeta_{h}^{n}, \zeta_{h}^{n}\right)_{L} \\
& -\frac{1}{2}(1-\gamma) \tau\left\|\theta_{h}^{n}\right\|_{a}^{2}-\gamma \tau\left(A_{h} \zeta_{h}^{n}, \theta_{h}^{n}\right)_{L}-\frac{1}{2}(1-\gamma) \tau\left\|\zeta_{h}^{n}\right\|_{a}^{2}+\tau\left(\alpha_{h}^{n}+\frac{1}{2} \beta_{h}^{n}, \theta_{h}^{n}\right)_{L}+\tau\left(\delta_{h}^{n}, \zeta_{h}^{n}\right)_{L}-\tau\left(\Psi_{h}^{n}, \zeta_{h}^{n}\right)_{L} .
\end{aligned}
$$

Rearranging the relation, completing the square in the three terms involving the $\|\cdot\|_{a}$-norm, and using the discrete stability (2.18) of $B_{h}$ yields the assertion. 


\subsection{Bound on source terms and basic energy estimate}

The goal of our second step is to bound the contributions of the source terms $\alpha_{h}^{n}, \beta_{h}^{n}, \delta_{h}^{n}$, and $\Psi_{h}^{n}$ on the right-hand side of the basic energy identity (4.1). To this purpose, we exploit the presence of the $|\cdot|_{S}^{2}$-terms and the $\|\cdot\|_{a}^{2}$-terms on the left-hand side (Lem. 4.3) so as to arrive at a basic energy estimate valid in all flow regimes and where the only term left to be bounded is the anti-dissipative term $\frac{1}{2}\left\|\xi_{h}^{n+1}-\zeta_{h}^{n}\right\|_{L}^{2}$ (Thm. 4.4). To fix the ideas, we assume $\gamma \in\left[\frac{1}{5}, \frac{2}{5}\right]$. A larger interval included in $\left(0, \frac{1}{2}\right)$ can be considered; this will only modify the numerical factors in front of the $\|\cdot\|_{a}^{2}$-terms. We introduce the quantity

$$
E_{h}^{n}:=t_{*}^{-1 / 2}\left\|\xi_{h}^{n}\right\|_{L}+\left\|\theta_{\pi}^{n}\right\|_{B *}+\left\|\theta_{\pi}^{n}\right\|_{A *}+\left\|\zeta_{\pi}^{n}\right\|_{B *}+\left\|\zeta_{\pi}^{n}\right\|_{A *}+C_{\Psi}^{n} \tau^{3 / 2},
$$

which collects, in addition to $t_{*}^{-1 / 2}\left\|\xi_{h}^{n}\right\|_{L}$, the space and time approximation errors. The contribution of the truncation error is already bounded in terms of the time step and the constant $C_{\Psi}^{n}$ defined by (3.14a); instead, we do not yet bound the space approximation errors $\theta_{\pi}^{n}$ and $\zeta_{\pi}^{n}$.

Lemma 4.3 (bound on source terms). Assume $\gamma \in\left[\frac{1}{5}, \frac{2}{5}\right]$ and $\mathrm{Co} \leq 1$. Then,

$$
\tau\left|\left(\alpha_{h}^{n}+\frac{1}{2} \beta_{h}^{n}, \theta_{h}^{n}\right)_{L}+\left(\delta_{h}^{n}, \zeta_{h}^{n}\right)_{L}-\left(\Psi_{h}^{n}, \zeta_{h}^{n}\right)_{L}\right| \leq \frac{1}{8} \tau\left|\theta_{h}^{n}\right|_{S}^{2}+\frac{1}{8} \tau\left|\zeta_{h}^{n}\right|_{S}^{2}+\frac{1}{40} \tau\left\|\theta_{h}^{n}\right\|_{a}^{2}+\frac{1}{80} \tau\left\|\zeta_{h}^{n}\right\|_{a}^{2}+C \tau\left(E_{h}^{n}\right)^{2}
$$

Proof. We first bound $\left\|\theta_{h}^{n}\right\|_{L}$ and $\left\|\zeta_{h}^{n}\right\|_{L}$. Taking the $L$-scalar product of (2.15a) with $\theta_{h}^{n}$ yields

$$
\left\|\theta_{h}^{n}\right\|_{L}^{2}+\gamma \tau\left\|\theta_{h}^{n}\right\|_{a}^{2}=\left(\xi_{h}^{n}, \theta_{h}^{n}\right)_{L}+\gamma \tau\left(A_{h} \theta_{\pi}^{n}, \theta_{h}^{n}\right)_{L} .
$$

Using (2.25) and the Cauchy-Schwarz inequality yields $\left\|\theta_{h}^{n}\right\|_{L}^{2}+\tau\left\|\theta_{h}^{n}\right\|_{a}^{2} \lesssim\left\|\xi_{h}^{n}\right\|_{L}\left\|\theta_{h}^{n}\right\|_{L}+\tau\left\|\theta_{\pi}^{n}\right\|_{A *}\left\|\theta_{h}^{n}\right\|_{A}$. Hence, using Young's inequality together with (2.17), we obtain

$$
\left\|\theta_{h}^{n}\right\|_{L}^{2}+\tau\left\|\theta_{h}^{n}\right\|_{a}^{2} \lesssim\left\|\xi_{h}^{n}\right\|_{L}^{2}+\tau\left\|\theta_{\pi}^{n}\right\|_{A *}^{2} .
$$

Taking now the $L$-scalar product of $(2.15 \mathrm{~b})$ with $\zeta_{h}^{n}$ yields

$$
\left\|\zeta_{h}^{n}\right\|_{L}^{2}+\gamma \tau\left\|\zeta_{h}^{n}\right\|_{a}^{2}=\left(\theta_{h}^{n}, \zeta_{h}^{n}\right)_{L}-\tau\left(B_{h} \theta_{h}^{n}, \zeta_{h}^{n}\right)_{L}-(1-3 \gamma) \tau\left(A_{h} \theta_{h}^{n}, \zeta_{h}^{n}\right)_{L}+\tau\left(\beta_{h}^{n}, \zeta_{h}^{n}\right)_{L}
$$

Using (2.23), the Cauchy-Schwarz inequality, and Co $\leq 1$, we infer $\tau\left|\left(B_{h} \theta_{h}^{n}, \zeta_{h}^{n}\right)_{L}\right| \lesssim\left\|\theta_{h}^{n}\right\|_{L}\left\|\zeta_{h}^{n}\right\|_{L}$. In addition, $\tau\left|\left(A_{h} \theta_{h}^{n}, \zeta_{h}^{n}\right)_{L}\right| \lesssim \tau\left\|\theta_{h}^{n}\right\|_{a}\left\|\zeta_{h}^{n}\right\|_{a}$ owing to (2.26) and (2.17), while using the boundedness (2.21) and (2.25) of $B_{h}$ and $A_{h}$, we infer

$$
\begin{aligned}
\tau\left|\left(\beta_{h}^{n}, \zeta_{h}^{n}\right)_{L}\right| & \lesssim \tau\left\|\theta_{\pi}^{n}\right\|_{B *}\left(\left|\zeta_{h}^{n}\right|_{S}+\sigma_{1}^{1 / 2}\left\|\zeta_{h}^{n}\right\|_{L}\right)+\tau\left(\left\|\theta_{\pi}^{n}\right\|_{A *}+\left\|\zeta_{\pi}^{n}\right\|_{A *}\right)\left\|\zeta_{h}^{n}\right\|_{A} \\
& \lesssim \tau^{1 / 2}\left\|\theta_{\pi}^{n}\right\|_{B *}\left\|\zeta_{h}^{n}\right\|_{L}+\tau\left(\left\|\theta_{\pi}^{n}\right\|_{A *}+\left\|\zeta_{\pi}^{n}\right\|_{A *}\right)\left\|\zeta_{h}^{n}\right\|_{A},
\end{aligned}
$$

where we have used $\tau \sigma_{1} \leq 1$, Co $\leq 1$, and (2.23). Hence,

$$
\left\|\zeta_{h}^{n}\right\|_{L}^{2}+\tau\left\|\zeta_{h}^{n}\right\|_{a}^{2} \lesssim\left\|\theta_{h}^{n}\right\|_{L}^{2}+\tau\left\|\theta_{h}^{n}\right\|_{a}^{2}+\tau\left(\left\|\theta_{\pi}^{n}\right\|_{B *}^{2}+\left\|\theta_{\pi}^{n}\right\|_{A *}^{2}+\left\|\zeta_{\pi}^{n}\right\|_{A *}^{2}\right)
$$

and accounting for (4.8) finally yields

$$
\left\|\zeta_{h}^{n}\right\|_{L}^{2}+\tau\left\|\zeta_{h}^{n}\right\|_{a}^{2} \lesssim\left\|\xi_{h}^{n}\right\|_{L}^{2}+\tau\left(\left\|\theta_{\pi}^{n}\right\|_{B *}^{2}+\left\|\theta_{\pi}^{n}\right\|_{A *}^{2}+\left\|\zeta_{\pi}^{n}\right\|_{A *}^{2}\right)
$$

We are now ready to bound the source terms. Since $\alpha_{h}^{n}=\gamma A_{h} \theta_{\pi}^{n}$ and $\left|\left(A_{h} \theta_{\pi}^{n}, \theta_{h}^{n}\right)_{L}\right| \lesssim\left\|\theta_{\pi}^{n}\right\|_{A *}\left\|\theta_{h}^{n}\right\|_{A} \lesssim$ $\left\|\theta_{\pi}^{n}\right\|_{A *}\left\|\theta_{h}^{n}\right\|_{a}$ owing to (2.25) and (2.17), we first obtain using Young's inequality

$$
\tau\left|\left(\alpha_{h}^{n}, \theta_{h}^{n}\right)_{L}\right| \leq \frac{1}{80} \tau\left\|\theta_{h}^{n}\right\|_{a}^{2}+C \tau\left\|\theta_{\pi}^{n}\right\|_{A *}^{2} \leq \frac{1}{80} \tau\left\|\theta_{h}^{n}\right\|_{a}^{2}+C \tau\left(E_{h}^{n}\right)^{2} .
$$


Similarly, recalling $\beta_{h}^{n}=B_{h} \theta_{\pi}^{n}+(1-3 \gamma) A_{h} \theta_{\pi}^{n}+\gamma A_{h} \zeta_{\pi}^{n}$ and using (2.21),

$$
\frac{1}{2} \tau\left|\left(\beta_{h}^{n}, \theta_{h}^{n}\right)_{L}\right| \leq \frac{1}{8} \tau\left(\left|\theta_{h}^{n}\right|_{S}^{2}+\sigma_{1}\left\|\theta_{h}^{n}\right\|_{L}^{2}\right)+\frac{1}{80} \tau\left\|\theta_{h}^{n}\right\|_{a}^{2}+C \tau\left(\left\|\theta_{\pi}^{n}\right\|_{B *}^{2}+\left\|\theta_{\pi}^{n}\right\|_{A *}^{2}+\left\|\zeta_{\pi}^{n}\right\|_{A *}^{2}\right) .
$$

Hence, using (4.8) to bound $\left\|\theta_{h}^{n}\right\|_{L}$ and since $\tau \leq t_{*} \leq \sigma_{1}^{-1}$, we infer

$$
\frac{1}{2} \tau\left|\left(\beta_{h}^{n}, \theta_{h}^{n}\right)_{L}\right| \leq \frac{1}{8} \tau\left|\theta_{h}^{n}\right|_{S}^{2}+\frac{1}{80} \tau\left\|\theta_{h}^{n}\right\|_{a}^{2}+C \tau\left(E_{h}^{n}\right)^{2} .
$$

Turning to $\delta_{h}^{n}$ and recalling that $\delta_{h}^{n}=\frac{1}{2} B_{h} \zeta_{\pi}^{n}+\frac{1}{2} \gamma A_{h} \theta_{\pi}^{n}+\frac{1}{2}(1-\gamma) A_{h} \zeta_{\pi}^{n}$ and proceeding as above, we infer

$$
\tau\left|\left(\delta_{h}^{n}, \zeta_{h}^{n}\right)_{L}\right| \leq \frac{1}{8} \tau\left|\zeta_{h}^{n}\right|_{S}^{2}+\frac{1}{160} \tau\left\|\zeta_{h}^{n}\right\|_{a}^{2}+C \tau\left(E_{h}^{n}\right)^{2} .
$$

Finally, concerning $\Psi_{h}^{n}$, we infer using (3.11), the Cauchy-Schwarz inequality, and Young's inequality (note in particular that $\left.\left(A x^{n}, \zeta_{h}^{n}\right)_{L}=\left(A_{h} x^{n}, \zeta_{h}^{n}\right)_{L} \lesssim\left\|x^{n}\right\|_{A *}\left\|\zeta_{h}^{n}\right\|_{A}\right)$,

$$
\begin{aligned}
\tau\left(\Psi_{h}^{n}, \zeta_{h}^{n}\right)_{L} & \leq \tau\left\|\tilde{\Psi}_{h}^{n}\right\|_{L}\left\|\zeta_{h}^{n}\right\|_{L}+\tau\left\|B x^{n}\right\|_{L}\left\|\zeta_{h}^{n}\right\|_{L}+\tau\left\|x^{n}\right\|_{A *}\left\|\zeta_{h}^{n}\right\|_{A} \\
& \leq \tau t_{*}\left(\left\|\tilde{\Psi}_{h}^{n}\right\|_{L}^{2}+\left\|B x^{n}\right\|_{L}^{2}\right)+\tau t_{*}^{-1}\left\|\zeta_{h}^{n}\right\|_{L}^{2}+\frac{1}{160} \tau\left\|\zeta_{h}^{n}\right\|_{a}^{2}+C \tau\left\|x^{n}\right\|_{A *}^{2}
\end{aligned}
$$

Using the bound (3.15b) on $\left\|\tilde{\Psi}^{n}\right\|_{L}+\left\|B x^{n}\right\|_{L}+t_{*}^{-1 / 2}\left\|x^{n}\right\|_{A *}$, we obtain

$$
\tau\left(\Psi_{h}^{n}, \zeta_{h}^{n}\right)_{L} \leq \frac{1}{160} \tau\left\|\zeta_{h}^{n}\right\|_{a}^{2}+\tau t_{*}^{-1}\left\|\zeta_{h}^{n}\right\|_{L}^{2}+C \tau\left(C_{\Psi}^{n}\right)^{2} \tau^{3},
$$

so that owing to the bound (4.9) on $\zeta_{h}^{n}, \tau \leq t_{*}$, and the definition of $E_{h}^{n}$,

$$
\tau\left(\Psi_{h}^{n}, \zeta_{h}^{n}\right)_{L} \leq \frac{1}{160} \tau\left\|\zeta_{h}^{n}\right\|_{a}^{2}+C \tau\left(E_{h}^{n}\right)^{2} .
$$

Collecting the bounds (4.10), (4.11), (4.12), and (4.13) yields the assertion.

Combining Lemmas 4.1 and 4.3 yields our basic energy estimate.

Theorem 4.4 (basic energy estimate). Assume $\gamma \in\left[\frac{1}{5}, \frac{2}{5}\right]$ and Co $\leq 1$. Then,

$$
\begin{aligned}
& \frac{1}{2}\left\|\xi_{h}^{n+1}\right\|_{L}^{2}-\frac{1}{2}\left\|\xi_{h}^{n}\right\|_{L}^{2}+\frac{1}{2}\left\|\theta_{h}^{n}-\xi_{h}^{n}\right\|_{L}^{2}+\frac{3}{8} \tau\left|\theta_{h}^{n}\right|_{S}^{2}+\frac{3}{8} \tau\left|\zeta_{h}^{n}\right|_{S}^{2}+\left(\frac{1}{2}-\gamma\right) \tau\left\|\theta_{h}^{n}\right\|_{a}^{2}+\frac{1}{20} \tau\left\|\zeta_{h}^{n}\right\|_{a}^{2}+\frac{1}{40} \tau\left\|\zeta_{h}^{n}+\theta_{h}^{n}\right\|_{a}^{2} \\
& \leq \frac{1}{2}\left\|\xi_{h}^{n+1}-\zeta_{h}^{n}\right\|_{L}^{2}+C \tau\left(E_{h}^{n}\right)^{2} .
\end{aligned}
$$

Proof. Using the energy identity (4.1) together with the fact that $\frac{1}{2}-\gamma \geq \frac{1}{10}$ and $\gamma \geq \frac{1}{5}$, and accounting for the bound (4.7) on the source terms yields

$$
\begin{aligned}
& \frac{1}{2}\left\|\xi_{h}^{n+1}\right\|_{L}^{2}-\frac{1}{2}\left\|\xi_{h}^{n}\right\|_{L}^{2}+\frac{1}{2}\left\|\theta_{h}^{n}-\xi_{h}^{n}\right\|_{L}^{2}+\frac{3}{8} \tau\left|\theta_{h}^{n}\right|_{S}^{2}+\frac{3}{8} \tau\left|\zeta_{h}^{n}\right|_{S}^{2}+\left(\frac{1}{2}-\gamma\right) \tau\left\|\theta_{h}^{n}\right\|_{a}^{2}+\frac{1}{10} \tau\left\|\zeta_{h}^{n}\right\|_{a}^{2}+\frac{1}{10} \tau\left\|\zeta_{h}^{n}+\theta_{h}^{n}\right\|_{a}^{2} \\
& \leq \frac{1}{2}\left\|\xi_{h}^{n+1}-\zeta_{h}^{n}\right\|_{L}^{2}+\frac{1}{40} \tau\left\|\theta_{h}^{n}\right\|_{a}^{2}+\frac{1}{80} \tau\left\|\zeta_{h}^{n}\right\|_{a}^{2}+C \tau\left(E_{h}^{n}\right)^{2} .
\end{aligned}
$$

Since the term involving $\left\|\theta_{h}^{n}\right\|_{a}^{2}$ on the left-hand side will be used later in a different context, we leave it as it stands and use instead the terms $\left\|\zeta_{h}^{n}\right\|_{a}^{2}$ and $\left\|\zeta_{h}^{n}+\theta_{h}^{n}\right\|_{a}^{2}$ on the left-hand side to absorb the two terms with the $\|\cdot\|_{a}$-norm on the right-hand side. We observe that

$$
\left\|\theta_{h}^{n}\right\|_{a}^{2}=\left\|\theta_{h}^{n}+\zeta_{h}^{n}-\zeta_{h}^{n}\right\|_{a}^{2} \leq \frac{3}{2}\left\|\zeta_{h}^{n}\right\|_{a}^{2}+3\left\|\zeta_{h}^{n}+\theta_{h}^{n}\right\|_{a}^{2}
$$

to infer the assertion.

The way to tackle the anti-dissipative term $\frac{1}{2}\left\|\xi_{h}^{n+1}-\zeta_{h}^{n}\right\|_{L}^{2}$ on the right-hand side of the basic energy estimate (4.14) depends on the flow regime and will be examined in the next two sections. 


\subsection{Stability and convergence: advection-dominated regime}

In this regime, we assume that $\mathrm{Pe} \geq 1$ and, as before to fix the ideas, that $\gamma \in\left[\frac{1}{5}, \frac{2}{5}\right]$. Taking a larger interval for $\gamma$ in $\left(0, \frac{1}{2}\right)$ is again possible, and this will only modify the numerical factors in the bound on the Courant number. In the advection-dominated regime, an important ingredient to bound the diffusion operator is that there is $C_{A}$ such that for all $v_{h} \in V_{h}$,

$$
\tau\left\|A_{h} v_{h}\right\|_{L} \leq C_{A}(\mathrm{Co} / \mathrm{Pe})^{1 / 2} \tau^{1 / 2}\left\|v_{h}\right\|_{A},
$$

since owing to (2.26), $\tau\left\|A_{h} v_{h}\right\|_{L} \lesssim \tau \mu^{1 / 2} h^{-1}\left\|v_{h}\right\|_{A}$ and $\tau^{1 / 2} \mu^{1 / 2} h^{-1}=(\mathrm{Co} / \mathrm{Pe})^{1 / 2}$.

Our first step is to control the anti-dissipative term $\frac{1}{2}\left\|\xi_{h}^{n+1}-\zeta_{h}^{n}\right\|_{L}^{2}$ on the right-hand side of the basic energy estimate (4.14). We use the following inverse inequality: there is $C_{i}$ such that for all $v_{h} \in V_{h}$,

$$
\left\|\nabla v_{h}\right\|_{L^{d}} \leq C_{i} h^{-1}\left\|v_{h}-\pi_{h}^{0} v_{h}\right\|_{L}
$$

where $\pi_{h}^{0}$ is defined in Lemma 2.4.

Lemma 4.5 (stability). Assume $\mathrm{Pe} \geq 1, \gamma \in\left[\frac{1}{5}, \frac{2}{5}\right]$, and $\mathrm{Co} \leq 1$. Assume further that

$$
\text { Co } \leq \min \left\{\frac{1}{2}\left(C_{i} C_{B}\right)^{-2 / 3}, \frac{1}{8} C_{S}^{-2}, \frac{5}{4} c_{a}\left(2 C_{i}+3\right)^{-2} C_{A}^{-2} \mathrm{Pe}\right\},
$$

recalling that $C_{B}$ and $C_{S}$ are defined in Lemma 2.4. Then,

$$
\frac{1}{2}\left\|\xi_{h}^{n+1}\right\|_{L}^{2}-\frac{1}{2}\left\|\xi_{h}^{n}\right\|_{L}^{2}+\frac{1}{2}\left\|\theta_{h}^{n}-\xi_{h}^{n}\right\|_{L}^{2}+\frac{1}{8} \tau\left|\theta_{h}^{n}\right|_{S}^{2}+\frac{1}{8} \tau\left|\zeta_{h}^{n}\right|_{S}^{2}+\frac{1}{20} c_{a} \tau\left\|\theta_{h}^{n}\right\|_{A}^{2}+\frac{1}{40} c_{a} \tau\left\|\zeta_{h}^{n}+\theta_{h}^{n}\right\|_{A}^{2} \lesssim \tau\left(E_{h}^{n}\right)^{2} .
$$

Proof. We start from the basic energy estimate (4.14) and observe that $\frac{1}{2}-\gamma \geq \frac{1}{10}$ to write

$$
\begin{aligned}
& \frac{1}{2}\left\|\xi_{h}^{n+1}\right\|_{L}^{2}-\frac{1}{2}\left\|\xi_{h}^{n}\right\|_{L}^{2}+\frac{1}{2}\left\|\theta_{h}^{n}-\xi_{h}^{n}\right\|_{L}^{2}+\frac{3}{8} \tau\left|\theta_{h}^{n}\right|_{S}^{2}+\frac{3}{8} \tau\left|\zeta_{h}^{n}\right|_{S}^{2}+\frac{1}{10} c_{a} \tau\left\|\theta_{h}^{n}\right\|_{A}^{2}+\frac{1}{20} c_{a} \tau\left\|\zeta_{h}^{n}\right\|_{A}^{2}+\frac{1}{40} c_{a} \tau\left\|\zeta_{h}^{n}+\theta_{h}^{n}\right\|_{A}^{2} \\
& \leq \frac{1}{2}\left\|\xi_{h}^{n+1}-\zeta_{h}^{n}\right\|_{L}^{2}+C \tau\left(E_{h}^{n}\right)^{2},
\end{aligned}
$$

where we have used (2.17) to replace the $\|\cdot\|_{a}$-norm by the $\|\cdot\|_{A}$-norm. Set $\eta_{h}^{n}:=\theta_{h}^{n}-\zeta_{h}^{n}$, so that by $(2.15 \mathrm{~b})$ and $(2.15 \mathrm{c})$,

$$
\xi_{h}^{n+1}-\zeta_{h}^{n}=\frac{1}{2} \tau B_{h} \eta_{h}^{n}+\left(\frac{1}{2}-2 \gamma\right) \tau A_{h} \theta_{h}^{n}-\left(\frac{1}{2}-\gamma\right) \tau A_{h} \zeta_{h}^{n}-\frac{1}{2} \tau \beta_{h}^{n}+\tau \delta_{h}^{n}-\tau \Psi_{h}^{n} .
$$

Using the triangle inequality and the bound $(2.20)$ on $B_{h}$ yields

$$
\begin{aligned}
\left\|\xi_{h}^{n+1}-\zeta_{h}^{n}\right\|_{L} \leq & \frac{1}{2} \sigma \tau\left\|\nabla \eta_{h}^{n}\right\|_{L^{d}}+\frac{1}{2} C_{S} \mathrm{Co}^{1 / 2} \tau^{1 / 2}\left|\eta_{h}^{n}\right|_{S}+\left|\frac{1}{2}-2 \gamma\right| \tau\left\|A_{h} \theta_{h}^{n}\right\|_{L}+\left(\frac{1}{2}-\gamma\right) \tau\left\|A_{h} \zeta_{h}^{n}\right\|_{L} \\
& +\tau\left(\frac{1}{2}\left\|\beta_{h}^{n}\right\|_{L}+\left\|\delta_{h}^{n}\right\|_{L}+\left\|\Psi^{n}\right\|_{L}\right) .
\end{aligned}
$$

The terms involving the discrete operator $A_{h}$ are bounded using (4.15), $\left|\frac{1}{2}-2 \gamma\right| \leq \frac{3}{10}$, and $\left(\frac{1}{2}-\gamma\right) \leq \frac{3}{10}$ yielding

$$
\left|\frac{1}{2}-2 \gamma\right| \tau\left\|A_{h} \theta_{h}^{n}\right\|_{L}+\left(\frac{1}{2}-\gamma\right) \tau\left\|A_{h} \zeta_{h}^{n}\right\|_{L} \leq \frac{3}{10} \tau^{1 / 2} C_{A}(\mathrm{Co} / \mathrm{Pe})^{1 / 2}\left(\left\|\theta_{h}^{n}\right\|_{A}+\left\|\zeta_{h}^{n}\right\|_{A}\right) .
$$

The contributions of $A_{h}$ to $\beta_{h}^{n}$ and $\delta_{h}^{n}$ are bounded using $(2.25)$ and $\tau^{1 / 2} \mu^{1 / 2} h^{-1}=(\mathrm{Co} / \mathrm{Pe})^{1 / 2} \leq 1$ so that

$$
\tau\left\|A_{h} \theta_{\pi}^{n}\right\|_{L}+\tau\left\|A_{h} \zeta_{\pi}^{n}\right\|_{L} \lesssim \tau \mu^{1 / 2} h^{-1}\left(\left\|\theta_{\pi}^{n}\right\|_{A *}+\left\|\zeta_{\pi}^{n}\right\|_{A *}\right) \leq \tau^{1 / 2} E_{h}^{n}
$$


The contributions of $B_{h}$ to $\beta_{h}^{n}$ and $\delta_{h}^{n}$ are bounded using (2.24) and Co $\leq 1$ so that

$$
\tau\left\|B_{h} \theta_{\pi}^{n}\right\|_{L}+\tau\left\|B_{h} \zeta_{\pi}^{n}\right\|_{L} \lesssim \tau^{1 / 2}\left(\left\|\theta_{\pi}^{n}\right\|_{B *}+\left\|\zeta_{\pi}^{n}\right\|_{B *}\right) \leq \tau^{1 / 2} E_{h}^{n}
$$

Hence,

$$
\tau\left\|\beta_{h}^{n}\right\|_{L}+\tau\left\|\delta_{h}^{n}\right\|_{L} \lesssim \tau^{1 / 2} E_{h}^{n}
$$

Finally,

$$
\tau\left\|\Psi_{h}^{n}\right\|_{L} \leq \tau\left\|\Psi^{n}\right\|_{L} \lesssim \tau \tilde{C}_{\Psi}^{n} \tau \leq \tau^{1 / 2} C_{\Psi}^{n} \tau^{3 / 2} \leq \tau^{1 / 2} E_{h}^{n},
$$

owing to the bound (3.15a) on $\left\|\Psi^{n}\right\|_{L}$ and the fact that $\tilde{C}_{\Psi}^{n} \leq C_{\Psi}^{n}$. As a result,

$$
\left\|\xi_{h}^{n+1}-\zeta_{h}^{n}\right\|_{L} \leq \frac{1}{2} \sigma \tau\left\|\nabla \eta_{h}^{n}\right\|_{L^{d}}+\frac{1}{2} C_{S} \mathrm{Co}^{1 / 2} \tau^{1 / 2}\left|\eta_{h}^{n}\right|_{S}+\frac{3}{10} C_{A}(\mathrm{Co} / \mathrm{Pe})^{1 / 2} \tau^{1 / 2}\left(\left\|\theta_{h}^{n}\right\|_{A}+\left\|\zeta_{h}^{n}\right\|_{A}\right)+C \tau^{1 / 2} E_{h}^{n}
$$

The next step is to control $\left\|\nabla \eta_{h}^{n}\right\|_{L^{d}}$. Let $\varsigma_{h}^{n}=\eta_{h}^{n}-\pi_{h}^{0} \eta_{h}^{n}$ and observe that

$$
\left\|\varsigma_{h}^{n}\right\|_{L}^{2}=\left(\eta_{h}^{n}, \varsigma_{h}^{n}\right)_{L}=\tau\left(B_{h} \theta_{h}^{n}, \varsigma_{h}^{n}\right)_{L}+(1-3 \gamma) \tau\left(A_{h} \theta_{h}^{n}, \varsigma_{h}^{n}\right)_{L}+\gamma \tau\left(A_{h} \zeta_{h}^{n}, \varsigma_{h}^{n}\right)_{L}-\tau\left(\beta_{h}^{n}, \varsigma_{h}^{n}\right)_{L},
$$

since $\eta_{h}^{n}=\tau B_{h} \theta_{h}^{n}+(1-3 \gamma) \tau A_{h} \theta_{h}^{n}+\gamma \tau A_{h} \zeta_{h}^{n}-\tau \beta_{h}^{n}$ owing to (2.15b). To bound the first term on the right-hand side, we use the bound $(2.22)$ on $B_{h}$ to infer

$$
\tau\left|\left(B_{h} \theta_{h}^{n}, \varsigma_{h}^{n}\right)_{L}\right| \leq C_{B} \mathrm{Co}^{1 / 2} \tau^{1 / 2}\left(\left|\theta_{h}^{n}\right|_{S}+\sigma_{1}^{1 / 2}\left\|\theta_{h}^{n}\right\|_{L}\right)\left\|\varsigma_{h}^{n}\right\|_{L}
$$

Furthermore, bounding the three other terms by the Cauchy-Schwarz inequality, using the fact that $\gamma \in\left[\frac{1}{5}, \frac{2}{5}\right]$ and the bound (4.15) for the terms involving the discrete operator $A_{h}$, and simplifying by $\left\|\varsigma_{h}^{n}\right\|_{L}$ yields

$$
\left\|\varsigma_{h}^{n}\right\|_{L} \leq C_{B} \mathrm{Co}^{1 / 2} \tau^{1 / 2}\left(\left|\theta_{h}^{n}\right|_{S}+\sigma_{1}^{1 / 2}\left\|\theta_{h}^{n}\right\|_{L}\right)+\frac{2}{5} C_{A}(\mathrm{Co} / \mathrm{Pe})^{1 / 2} \tau^{1 / 2}\left(\left\|\theta_{h}^{n}\right\|_{A}+\left\|\zeta_{h}^{n}\right\|_{A}\right)+\tau\left\|\beta_{h}^{n}\right\|_{L},
$$

so that using the bound (4.8) on $\left\|\theta_{h}^{n}\right\|_{L}, \tau \sigma_{1} \leq 1$, and (4.20) to bound $\tau\left\|\beta_{h}^{n}\right\|_{L}$ leads to

$$
\left\|\varsigma_{h}^{n}\right\|_{L} \leq C_{B} \mathrm{Co}^{1 / 2} \tau^{1 / 2}\left|\theta_{h}^{n}\right|_{S}+\frac{2}{5} C_{A}(\mathrm{Co} / \mathrm{Pe})^{1 / 2} \tau^{1 / 2}\left(\left\|\theta_{h}^{n}\right\|_{A}+\left\|\zeta_{h}^{n}\right\|_{A}\right)+C \tau^{1 / 2} E_{h}^{n}
$$

Thus, using the inverse inequality (4.16), we obtain

$$
\begin{aligned}
\sigma \tau\left\|\nabla \eta_{h}^{n}\right\|_{L^{d}} & \leq C_{i} \sigma \tau h^{-1}\left\|\varsigma_{h}^{n}\right\|_{L}=C_{i} \mathrm{Co}\left\|\varsigma_{h}^{n}\right\|_{L} \\
& \leq C_{i} C_{B} \mathrm{Co}^{3 / 2} \tau^{1 / 2}\left|\theta_{h}^{n}\right|_{S}+\frac{2}{5} C_{i} C_{A} \mathrm{Co}(\mathrm{Co} / \mathrm{Pe})^{1 / 2} \tau^{1 / 2}\left(\left\|\theta_{h}^{n}\right\|_{A}+\left\|\zeta_{h}^{n}\right\|_{A}\right)+C \tau^{1 / 2} E_{h}^{n}
\end{aligned}
$$

Substituting back into (4.22), re-arranging terms, and since Co $\leq 1$, we infer

$$
\begin{aligned}
\left\|\xi_{h}^{n+1}-\zeta_{h}^{n}\right\|_{L} \leq & \frac{1}{2} C_{i} C_{B} \mathrm{Co}^{3 / 2} \tau^{1 / 2}\left|\theta_{h}^{n}\right|_{S}+\frac{1}{2} C_{S} \mathrm{Co}^{1 / 2} \tau^{1 / 2}\left|\theta_{h}^{n}-\zeta_{h}^{n}\right|_{S} \\
& +\left(\frac{1}{5} C_{i}+\frac{3}{10}\right) C_{A}(\mathrm{Co} / \mathrm{Pe})^{1 / 2} \tau^{1 / 2}\left(\left\|\theta_{h}^{n}\right\|_{A}+\left\|\zeta_{h}^{n}\right\|_{A}\right)+C \tau^{1 / 2} E_{h}^{n}
\end{aligned}
$$

Let $\chi_{1}:=32^{-1 / 2}$ and $\chi_{2}:=80^{-1 / 2}$. Then, owing to the assumption (4.17) on the Courant number, the above inequality becomes

$$
\left\|\xi_{h}^{n+1}-\zeta_{h}^{n}\right\|_{L} \leq \chi_{1} \tau^{1 / 2}\left(\left|\theta_{h}^{n}\right|_{S}+\left|\theta_{h}^{n}-\zeta_{h}^{n}\right|_{S}\right)+\chi_{2} c_{a}^{1 / 2} \tau^{1 / 2}\left(\left\|\theta_{h}^{n}\right\|_{A}+\left\|\zeta_{h}^{n}\right\|_{A}\right)+C \tau^{1 / 2} E_{h}^{n}
$$

Since $\left|\theta_{h}^{n}\right|_{S}+\left|\theta_{h}^{n}-\zeta_{h}^{n}\right|_{S} \leq 2\left(\left|\theta_{h}^{n}\right|_{S}+\left|\zeta_{h}^{n}\right|_{S}\right)$, squaring the above bound, and using that $\frac{1}{2}(a+b+c)^{2} \leq a^{2}+2 b^{2}+2 c^{2}$ where $a, b$, and $c$ denote the three addends on the right-hand side of the above equation yields

$$
\frac{1}{2}\left\|\xi_{h}^{n+1}-\zeta_{h}^{n}\right\|_{L}^{2} \leq 8 \chi_{1}^{2} \tau\left(\left|\theta_{h}^{n}\right|_{S}^{2}+\left|\zeta_{h}^{n}\right|_{S}^{2}\right)+4 \chi_{2}^{2} c_{a} \tau\left(\left\|\theta_{h}^{n}\right\|_{A}^{2}+\left\|\zeta_{h}^{n}\right\|_{A}^{2}\right)+C \tau E_{h}^{n} .
$$

Finally, observing that $8 \chi_{1}^{2}=\frac{1}{4}$ and $4 \chi_{2}^{2}=\frac{1}{20}$ yields the assertion. 
Remark 4.6 (purely advective case). In the purely advective case $(\mu=0)$, the third argument in the bound (4.17) on the Courant number can be dropped, leading to the bound derived in [8].

Remark 4.7 (parabolic CFL restriction). In the advection-dominated regime, there holds $\tau \mu h^{-2}=\mathrm{CoPe}^{-1} \leq$ 1 , which is a parabolic CFL restriction on the time step. In particular, this property has been used in the proof of Lemma 4.5 to control the terms with the discrete operator $A_{h}$ using (4.15). We stress that this property is not used in the diffusion-dominated regime, where it will be too restrictive. We also notice that this property is compatible with (2.3) since, in all cases, $\mu \leq \bar{\mu}$; actually, in the advection-dominated regime, there holds in general $\bar{\mu}=\sigma^{2} t_{*} \gg \mu$.

We can now derive our main convergence result in the advection-dominated regime.

Theorem 4.8 (convergence in L-norm). With the basic assumptions stated in Section 2.1, assume $\mathrm{Pe} \geq 1$, take $\gamma \in\left[\frac{1}{5}, \frac{2}{5}\right]$, and assume the bound (4.17) on the Courant number. Then,

$$
\left\|u^{N}-u_{h}^{N}\right\|_{L} \lesssim C_{\mathrm{tim}} \tau^{3 / 2}+C_{\mathrm{spc}} \sigma^{1 / 2} h^{3 / 2}
$$

where $C_{\mathrm{tim}}^{2}=\sum_{n=0}^{N-1} \tau\left(C_{\Psi}^{n}\right)^{2}$ with $C_{\Psi}^{n}$ defined by (3.14a) and $C_{\mathrm{spc}}^{2}=\sum_{n=0}^{N-1} \tau\left(\left(\tilde{K}_{2}^{n}\right)^{2}+\left(\sigma^{-1} K_{w-u}^{n}\right)^{2}\right)$ with $\tilde{K}_{2}^{n}$ and $K_{w-u}^{n}$ defined in Lemmas 3.2 and 3.3 respectively.

Proof. Using the stability result of Lemma 4.5, we sum over $n$, discard the dissipative terms on the left-hand side, and use a discrete Gronwall lemma to eliminate the contribution of $\left\|\xi_{h}^{n}\right\|_{L}^{2}$ in $E_{h}^{n}$. This yields

$$
\left\|\xi_{h}^{N}\right\|_{L}^{2} \lesssim \sum_{n=0}^{N-1} \tau\left(\left\|\theta_{\pi}^{n}\right\|_{B *}^{2}+\left\|\zeta_{\pi}^{n}\right\|_{B *}^{2}+\left\|\theta_{\pi}^{n}\right\|_{A *}^{2}+\left\|\zeta_{\pi}^{n}\right\|_{A *}^{2}+\left(C_{\Psi}^{n}\right)^{2} \tau^{3}\right) .
$$

To bound the terms with $\theta_{\pi}^{n}$ and $\zeta_{\pi}^{n}$, we use the result of Lemma 3.8, and the fact that $\mu^{1 / 2} \leq \sigma^{1 / 2} h^{1 / 2}$ since $\mathrm{Pe} \geq 1$ and $\tau^{1 / 2} h K_{w-u}^{n} \leq \sigma^{1 / 2} h^{3 / 2}\left(\sigma^{-1} K_{w-u}^{n}\right)$ since $\mathrm{Co} \leq 1$. This yields $\left\|\xi_{h}^{N}\right\|_{L} \lesssim C_{\mathrm{tim}} \tau^{3 / 2}+C_{\mathrm{spc}} \sigma^{1 / 2} h^{3 / 2}$, and we conclude using the triangle inequality.

The convergence result of Theorem 4.8 can be completed by showing additionally the convergence in the $\|\cdot\|_{A}$-norm. The proof is postponed to Section 7.1 .

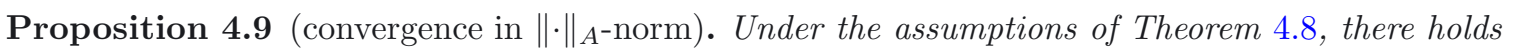

$$
\left(\tau \sum_{n=1}^{N}\left\|u^{n}-u_{h}^{n}\right\|_{A}^{2}\right)^{1 / 2} \lesssim C_{\mathrm{tim}} \tau^{3 / 2}+C_{\mathrm{spc}} \sigma^{1 / 2} h^{3 / 2}
$$

\subsection{Stability and convergence: diffusion-dominated regime}

In this regime, we assume $\mathrm{Pe} \leq 1$. We derive three intermediate stability results. First (Lem. 4.10), we tighten the basic energy estimate $(4.14)$ by achieving additional control on the increment $\left\|\theta_{h}^{n}-\zeta_{h}^{n}\right\|_{L}^{2}$. Then (Lem. 4.12), we bound the anti-dissipative term $\frac{1}{2}\left\|\xi_{h}^{n+1}-\zeta_{h}^{n}\right\|_{L}^{2}$. Finally (Lem. 4.13), we achieve additional control on $\tau\left\|\xi_{h}^{n+1}\right\|_{A}^{2}$. For our first step, it is sufficient that $\gamma \in\left(\frac{1}{4}, \frac{2}{5}\right]$; the minimal threshold on $\gamma$ serves to obtain only positive factors on the left-hand side of the new energy estimate (4.25). For our second and third steps, we need the parameter $\gamma$ to be sufficiently close to $\gamma_{*}=1-\frac{1}{\sqrt{2}} \simeq 0.293$. For simplicity, we assume $\gamma=\gamma_{*}$ and postpone to Remark 4.15 the discussion when $\gamma$ slightly deviates from $\gamma_{*}$, as motivated for instance by finite arithmetic precision.

In the diffusion-dominated regime, an important ingredient to bound the operator $B_{h}$ is that there is $C_{B A}$ such that for all $v_{h} \in V_{h}$,

$$
\tau\left\|B_{h} v_{h}\right\|_{L} \leq C_{B A} \tau \sigma \mu^{-1 / 2}\left\|v_{h}\right\|_{A}=C_{B A}(\mathrm{CoPe})^{1 / 2} \tau^{1 / 2}\left\|v_{h}\right\|_{A},
$$

since owing to the definition of the $\|\cdot\|_{A^{-n o r m}}$, $\left\|\nabla v_{h}\right\|_{L^{d}} \leq \mu^{-1 / 2}\left\|v_{h}\right\|_{A}$, while a discrete trace inequality yields $\left|v_{h}\right|_{S} \lesssim\left(\frac{\sigma h}{\mu}\right)^{1 / 2}\left\|v_{h}\right\|_{A}$, so that (4.24) results from $(2.20)$ and $\tau^{1 / 2} \sigma \mu^{-1 / 2}=(\mathrm{CoPe})^{1 / 2}$. 
Lemma 4.10. Assume $\gamma \in\left(\frac{1}{4}, \frac{2}{5}\right]$. Assume $\mathrm{Co} \leq \min \left(1, \frac{1}{30} c_{a} C_{B A}^{-2} \mathrm{Pe}^{-1}\right)$. Then,

$$
\begin{aligned}
& \frac{1}{2}\left\|\xi_{h}^{n+1}\right\|_{L}^{2}-\frac{1}{2}\left\|\xi_{h}^{n}\right\|_{L}^{2}+\frac{1}{2}\left\|\theta_{h}^{n}-\xi_{h}^{n}\right\|_{L}^{2}+\frac{3}{8}\left\|\theta_{h}^{n}-\zeta_{h}^{n}\right\|_{L}^{2}+\frac{1}{8} \tau\left|\theta_{h}^{n}\right|_{S}^{2}+\frac{1}{8} \tau\left|\zeta_{h}^{n}\right|_{S}^{2}+\frac{1}{8}\left(\frac{1}{2}-\gamma\right) c_{a} \tau\left\|\theta_{h}^{n}\right\|_{A}^{2} \\
& +\frac{1}{8} c_{a} \tau\left\|\zeta_{h}^{n}\right\|_{A}^{2}+\frac{3}{4}\left(\gamma-\frac{1}{4}\right) c_{a} \tau\left\|\theta_{h}^{n}-\zeta_{h}^{n}\right\|_{A}^{2}+\frac{1}{40} c_{a} \tau\left\|\zeta_{h}^{n}+\theta_{h}^{n}\right\|_{A}^{2} \leq \frac{1}{2}\left\|\xi_{h}^{n+1}-\zeta_{h}^{n}\right\|_{L}^{2}+C \tau\left(E_{h}^{n}\right)^{2} .
\end{aligned}
$$

Proof. We first observe that (2.15b) implies

$$
\theta_{h}^{n}-\zeta_{h}^{n}=\tau B_{h} \theta_{h}^{n}+(1-3 \gamma) \tau A_{h} \theta_{h}^{n}+\gamma \tau A_{h} \zeta_{h}^{n}-\tau \beta_{h}^{n},
$$

and re-arranging terms leads to

$$
\theta_{h}^{n}-\zeta_{h}^{n}=\tau B_{h} \theta_{h}^{n}-\left(\gamma-\frac{1}{2}\right) \tau A_{h}\left(\theta_{h}^{n}+\zeta_{h}^{n}\right)-\left(2 \gamma-\frac{1}{2}\right) \tau A_{h}\left(\theta_{h}^{n}-\zeta_{h}^{n}\right)-\tau \beta_{h}^{n} .
$$

Taking the $L$-scalar product with $\theta_{h}^{n}-\zeta_{h}^{n}$ and using the symmetry of $a_{h}$ yields

$$
\left\|\theta_{h}^{n}-\zeta_{h}^{n}\right\|_{L}^{2}=\tau\left(B_{h} \theta_{h}^{n}, \theta_{h}^{n}-\zeta_{h}^{n}\right)_{L}-\left(\gamma-\frac{1}{2}\right) \tau\left(\left\|\theta_{h}^{n}\right\|_{a}^{2}-\left\|\zeta_{h}^{n}\right\|_{a}^{2}\right)-\left(2 \gamma-\frac{1}{2}\right) \tau\left\|\theta_{h}^{n}-\zeta_{h}^{n}\right\|_{a}^{2}-\tau\left(\beta_{h}^{n}, \theta_{h}^{n}-\zeta_{h}^{n}\right)_{L}
$$

Since $\tau\left(B_{h} \theta_{h}^{n}, \theta_{h}^{n}-\zeta_{h}^{n}\right)_{L} \leq \frac{1}{2} \tau^{2}\left\|B_{h} \theta_{h}^{n}\right\|_{L}^{2}+\frac{1}{2}\left\|\theta_{h}^{n}-\zeta_{h}^{n}\right\|_{L}^{2}$, this yields, re-arranging terms,

$$
\frac{1}{2}\left\|\theta_{h}^{n}-\zeta_{h}^{n}\right\|_{L}^{2}+\left(\gamma-\frac{1}{2}\right) \tau\left(\left\|\theta_{h}^{n}\right\|_{a}^{2}-\left\|\zeta_{h}^{n}\right\|_{a}^{2}\right)+\left(2 \gamma-\frac{1}{2}\right) \tau\left\|\theta_{h}^{n}-\zeta_{h}^{n}\right\|_{a}^{2} \leq \frac{1}{2} \tau^{2}\left\|B_{h} \theta_{h}^{n}\right\|_{L}^{2}-\tau\left(\beta_{h}^{n}, \theta_{h}^{n}-\zeta_{h}^{n}\right)_{L} .
$$

The idea is now to combine (4.26) with (4.14) so as to absorb the positive term $\frac{1}{2} \tau^{2}\left\|B_{h} \theta_{h}^{n}\right\|_{L}^{2}$ by dissipative terms on the left-hand side. To this purpose, we multiply (4.26) by a real number $\alpha \in(0,1)$ and sum the resulting estimate to (4.14). To fix the ideas, we take $\alpha=\frac{3}{4}$ yielding

$$
\begin{aligned}
& \frac{1}{2}\left\|\xi_{h}^{n+1}\right\|_{L}^{2}-\frac{1}{2}\left\|\xi_{h}^{n}\right\|_{L}^{2}+\frac{1}{2}\left\|\theta_{h}^{n}-\xi_{h}^{n}\right\|_{L}^{2}+\frac{3}{8}\left\|\theta_{h}^{n}-\zeta_{h}^{n}\right\|_{L}^{2}+\frac{3}{8} \tau\left|\theta_{h}^{n}\right|_{S}^{2}+\frac{3}{8} \tau\left|\zeta_{h}^{n}\right|_{S}^{2} \\
& +\frac{1}{4}\left(\frac{1}{2}-\gamma\right) c_{a} \tau\left\|\theta_{h}^{n}\right\|_{A}^{2}+\frac{1}{8} c_{a} \tau\left\|\zeta_{h}^{n}\right\|_{A}^{2}+\frac{3}{4}\left(2 \gamma-\frac{1}{2}\right) c_{a} \tau\left\|\theta_{h}^{n}-\zeta_{h}^{n}\right\|_{A}^{2}+\frac{1}{40} c_{a} \tau\left\|\zeta_{h}^{n}+\theta_{h}^{n}\right\|_{A}^{2} \\
& \leq \frac{1}{2}\left\|\xi_{h}^{n+1}-\zeta_{h}^{n}\right\|_{L}^{2}+\frac{3}{8} \tau^{2}\left\|B_{h} \theta_{h}^{n}\right\|_{L}^{2}-\frac{3}{4} \tau\left(\beta_{h}^{n}, \theta_{h}^{n}-\zeta_{h}^{n}\right)_{L}+C \tau\left(E_{h}^{n}\right)^{2},
\end{aligned}
$$

where we have used the discrete stability (2.17) of $A_{h}$ to substitute the $\|\cdot\|_{a}$-norms by $\|\cdot\|_{A}$-norms on the lefthand side and the fact that $\frac{1}{2}-\gamma \geq \frac{1}{10}$ to simplify the term with $\left\|\zeta_{h}^{n}\right\|_{A}^{2}$. Using (4.24) and the assumption on the Courant number yields

$$
\frac{3}{8} \tau^{2}\left\|B_{h} \theta_{h}^{n}\right\|_{L}^{2} \leq \frac{1}{80} c_{a} \tau\left\|\theta_{h}^{n}\right\|_{A}^{2}=\frac{1}{8}\left\{\min _{\gamma \in\left(\frac{1}{4}, \frac{2}{5}\right]}\left(\frac{1}{2}-\gamma\right)\right\} c_{a} \tau\left\|\theta_{h}^{n}\right\|_{A}^{2},
$$

so that this term can be absorbed using half of the $\left\|\theta_{h}^{n}\right\|_{A^{2}}^{2}$ term on the left-hand side of the above energy estimate. Finally, we bound $\frac{3}{4} \tau\left(\beta_{h}^{n}, \theta_{h}^{n}-\zeta_{h}^{n}\right)_{L}$. We obtain using the boundedness (2.21) and (2.25) of $B_{h}$ and $A_{h}$,

$$
\frac{3}{4} \tau\left|\left(\beta_{h}^{n}, \theta_{h}^{n}-\zeta_{h}^{n}\right)_{L}\right| \lesssim \tau\left\|\theta_{\pi}^{n}\right\|_{B *}\left(\left|\theta_{h}^{n}-\zeta_{h}^{n}\right|_{S}+\sigma_{1}^{1 / 2}\left\|\theta_{h}^{n}-\zeta_{h}^{n}\right\|_{L}\right)+\tau\left(\left\|\theta_{\pi}^{n}\right\|_{A *}+\left\|\zeta_{\pi}^{n}\right\|_{A *}\right)\left\|\theta_{h}^{n}-\zeta_{h}^{n}\right\|_{A}
$$


The first term is bounded as

$$
\begin{aligned}
\tau\left\|\theta_{\pi}^{n}\right\|_{B *}\left(\left|\theta_{h}^{n}-\zeta_{h}^{n}\right|_{S}+\sigma_{1}^{1 / 2}\left\|\theta_{h}^{n}-\zeta_{h}^{n}\right\|_{L}\right) & \leq \frac{1}{4} \tau\left(\left|\theta_{h}^{n}\right|_{S}^{2}+\left|\zeta_{h}^{n}\right|_{S}^{2}\right)+C \tau\left(\left\|\theta_{\pi}^{n}\right\|_{B *}^{2}+\sigma_{1}\left\|\theta_{h}^{n}\right\|_{L}^{2}+\sigma_{1}\left\|\zeta_{h}^{n}\right\|_{L}^{2}\right) \\
& \leq \frac{1}{4} \tau\left(\left|\theta_{h}^{n}\right|_{S}^{2}+\left|\zeta_{h}^{n}\right|_{S}^{2}\right)+C \tau\left(E_{h}^{n}\right)^{2}
\end{aligned}
$$

where we have used $\tau \sigma_{1} \leq 1$ and the bounds (4.8) and (4.9) on $\left\|\theta_{h}^{n}\right\|_{L}$ and $\left\|\zeta_{h}^{n}\right\|_{L}$. For the second term,

$$
\tau\left(\left\|\theta_{\pi}^{n}\right\|_{A *}+\left\|\zeta_{\pi}^{n}\right\|_{A *}\right)\left\|\theta_{h}^{n}-\zeta_{h}^{n}\right\|_{A} \leq \frac{3}{4}\left(\gamma-\frac{1}{4}\right) \tau\left\|\theta_{h}^{n}-\zeta_{h}^{n}\right\|_{A}^{2}+C \tau\left(\left\|\theta_{\pi}^{n}\right\|_{A *}^{2}+\left\|\zeta_{\pi}^{n}\right\|_{A *}^{2}\right)
$$

Collecting the above estimates yields the assertion.

Our second step aims at controlling the anti-dissipative term $\frac{1}{2}\left\|\xi_{h}^{n+1}-\zeta_{h}^{n}\right\|_{L}^{2}$ on the right-hand side of the energy estimate (4.25). To this purpose, it is useful to reformulate the last step (2.15c) of the error equation without using the discrete operator $A_{h}$. We simply state the result, since the proof amounts to a direct verification.

Lemma 4.11 (reformulation of last step without $\left.A_{h}\right)$. Let $\omega_{1}:=\gamma^{-1}\left(\frac{1}{2}-\gamma\right)$ and $\omega_{2}:=\frac{1}{2 \gamma^{2}}\left(-1+4 \gamma-2 \gamma^{2}\right)$. Then,

$$
\xi_{h}^{n+1}-\zeta_{h}^{n}=\omega_{1}\left(\zeta_{h}^{n}-\theta_{h}^{n}\right)+\omega_{2}\left(\theta_{h}^{n}-\xi_{h}^{n}\right)-\frac{1}{2} \tau B_{h}\left(\zeta_{h}^{n}-\theta_{h}^{n}\right)+\omega_{1} \tau B_{h} \theta_{h}^{n}-\tau \Xi_{h}^{n}-\tau \Psi_{h}^{n},
$$

where

$$
\Xi_{h}^{n}:=-\frac{1}{2} B_{h}\left(\zeta_{\pi}^{n}-\theta_{\pi}^{n}\right)+\omega_{1} B_{h} \theta_{\pi}^{n} .
$$

In what follows, we assume $\gamma=\gamma_{*}$. An important fact used hereafter is that $\omega_{2}\left(\gamma_{*}\right)=0$, thereby zeroing out the contribution of $\xi_{h}^{n}$ on the right-hand side of (4.27). We are now ready to bound the anti-dissipative term. Note that we tighten the assumption on the Courant number with respect to Lemma 4.10.

Lemma 4.12. Assume $\gamma=\gamma_{*}$ and

$$
\mathrm{Co} \leq \min \left(1, \frac{1}{180} c_{a} C_{B A}^{-2} \mathrm{Pe}^{-1}\right)
$$

Then,

$$
\frac{1}{2}\left\|\xi_{h}^{n+1}\right\|_{L}^{2}-\frac{1}{2}\left\|\xi_{h}^{n}\right\|_{L}^{2}+\frac{1}{8} \tau\left|\theta_{h}^{n}\right|_{S}^{2}+\frac{1}{8} \tau\left|\zeta_{h}^{n}\right|_{S}^{2}+\frac{1}{80} c_{a} \tau\left\|\theta_{h}^{n}\right\|_{A}^{2}+\frac{1}{8} c_{a} \tau\left\|\zeta_{h}^{n}\right\|_{A}^{2}+\frac{1}{40} c_{a} \tau\left\|\zeta_{h}^{n}+\theta_{h}^{n}\right\|_{A}^{2} \lesssim \tau\left(E_{h}^{n}\right)^{2} .
$$

Proof. We start from the result of Lemma 4.11. Observing that $\omega_{1}=\frac{1}{\sqrt{2}}$ and $\omega_{2}=0$ for $\gamma=\gamma_{*}$ and setting

$$
X_{h}^{n}=-\frac{1}{2} B_{h}\left(\zeta_{h}^{n}-\theta_{h}^{n}\right)+\frac{1}{\sqrt{2}} B_{h} \theta_{h}^{n}-\Xi_{h}^{n}-\Psi_{h}^{n},
$$

where $\Xi_{h}^{n}$ is defined by $(4.28)$, we infer

$$
\xi_{h}^{n+1}-\zeta_{h}^{n}=\frac{1}{\sqrt{2}}\left(\zeta_{h}^{n}-\theta_{h}^{n}\right)+\tau X_{h}^{n}
$$

This yields for positive real number $\epsilon, \frac{1}{2}\left\|\xi_{h}^{n+1}-\zeta_{h}^{n}\right\|_{L}^{2} \leq \frac{1}{2}\left(1+\epsilon^{-1}\right) \tau^{2}\left\|X_{h}^{n}\right\|_{L}^{2}+\frac{1}{4}(1+\epsilon)\left\|\zeta_{h}^{n}-\theta_{h}^{n}\right\|_{L}^{2}$. Choosing $\epsilon=\frac{1}{2}$, we infer

$$
\frac{1}{2}\left\|\xi_{h}^{n+1}-\zeta_{h}^{n}\right\|_{L}^{2} \leq \frac{3}{2} \tau^{2}\left\|X_{h}^{n}\right\|_{L}^{2}+\frac{3}{8}\left\|\zeta_{h}^{n}-\theta_{h}^{n}\right\|_{L}^{2} .
$$


We now bound the term $\left\|X_{h}^{n}\right\|_{L}^{2}$. Since $\frac{1}{3}(a+b+c)^{2} \leq a^{2}+b^{2}+c^{2}$ for real numbers $a, b$, and $c$, we obtain using (4.24),

$$
\begin{aligned}
\frac{1}{3} \tau^{2}\left\|X_{h}^{n}\right\|_{L}^{2} & \leq \frac{1}{4} \tau^{2}\left\|B_{h}\left(\zeta_{h}^{n}-\theta_{h}^{n}\right)\right\|_{L}^{2}+\frac{1}{2} \tau^{2}\left\|B_{h} \theta_{h}^{n}\right\|_{L}^{2}+\tau^{2}\left\|\Xi_{h}^{n}+\Psi_{h}^{n}\right\|_{L}^{2} \\
& \leq C_{B A}^{2}(\mathrm{CoPe}) \tau\left(\frac{1}{4}\left\|\zeta_{h}^{n}-\theta_{h}^{n}\right\|_{A}^{2}+\frac{1}{2}\left\|\theta_{h}^{n}\right\|_{A}^{2}\right)+\tau^{2}\left\|\Xi_{h}^{n}+\Psi_{h}^{n}\right\|_{L}^{2} .
\end{aligned}
$$

Owing to (2.24) and Co $\leq 1, \tau\left\|\Xi_{h}^{n}\right\|_{L} \lesssim \tau^{1 / 2} E_{h}^{n}$ and recalling $\tau\left\|\Psi_{h}^{n}\right\|_{L} \lesssim \tau^{1 / 2} E_{h}^{n}$ from (4.21), we obtain

$$
\frac{1}{3} \tau^{2}\left\|X_{h}^{n}\right\|_{L}^{2} \leq C_{B A}^{2}(\mathrm{CoPe}) \tau\left(\frac{1}{4}\left\|\zeta_{h}^{n}-\theta_{h}^{n}\right\|_{A}^{2}+\frac{1}{2}\left\|\theta_{h}^{n}\right\|_{A}^{2}\right)+C \tau\left(E_{h}^{n}\right)^{2} .
$$

Owing to the assumption on the Courant number,

$$
3 \frac{3}{2} \frac{1}{4} C_{B A}^{2} c_{a}^{-1}(\mathrm{CoPe}) \leq \frac{1}{160} \leq \frac{3}{4}\left(\gamma_{*}-\frac{1}{4}\right), \quad 3 \frac{3}{2} \frac{1}{2} C_{B A}^{2} c_{a}^{-1}(\mathrm{CoPe}) \leq \frac{1}{80} \leq \frac{1}{16}\left(\frac{1}{2}-\gamma_{*}\right) .
$$

As a result,

$$
\frac{1}{2}\left\|\xi_{h}^{n+1}-\zeta_{h}^{n}\right\|_{L}^{2} \leq \frac{3}{4}\left(\gamma_{*}-\frac{1}{4}\right) c_{a} \tau\left\|\zeta_{h}^{n}-\theta_{h}^{n}\right\|_{A}^{2}+\frac{1}{16}\left(\frac{1}{2}-\gamma_{*}\right) c_{a} \tau\left\|\theta_{h}^{n}\right\|_{A}^{2}+\frac{3}{8}\left\|\zeta_{h}^{n}-\theta_{h}^{n}\right\|_{L}^{2}+C \tau\left(E_{h}^{n}\right)^{2} .
$$

Using this estimate in (4.25) yields the assertion since $\frac{1}{16}\left(\frac{1}{2}-\gamma_{*}\right) \geq \frac{1}{80}$.

We can now proceed to our third and final step in the stability analysis. Our goal is to infer a control on $\tau\left\|\xi_{h}^{n+1}\right\|_{A}^{2}$ from the control on $\tau\left\|\theta_{h}^{n}\right\|_{A}^{2}$ and $\tau\left\|\zeta_{h}^{n}\right\|_{A}^{2}$ achieved in (4.30). This will require replacing the quantity $E_{h}^{n}$ by

$$
\tilde{E}_{h}^{n}:=t_{*}^{-1 / 2}\left\|\xi_{h}^{n}\right\|_{L}+\left\|\theta_{\pi}^{n}\right\|_{B *}+\left\|\theta_{\pi}^{n}\right\|_{A *}+\left\|\zeta_{\pi}^{n}\right\|_{B *}+\left\|\zeta_{\pi}^{n}\right\|_{A *}+\operatorname{Pe}^{-1 / 2}\left(\left|\theta_{\pi}^{n}\right|_{S}+\left|\zeta_{\pi}^{n}\right|_{S}\right)+t_{*}^{1 / 2} \tilde{C}_{\Psi}^{n} \tau .
$$

The definition of $\tilde{E}_{h}^{n}$ entails two modifications with respect to $E_{h}^{n}$. Firstly, the term $\mathrm{Pe}^{-1 / 2}\left(\left|\theta_{\pi}^{n}\right|_{S}+\left|\zeta_{\pi}^{n}\right|_{S}\right)$ has been added; this change will not modify the convergence rate in space with respect to that of the $\|\cdot\|_{A^{-}}$and $\|\cdot\|_{B *}$-norms of $\theta_{\pi}^{n}$ and $\zeta_{\pi}^{n}$. Secondly, and more importantly, the time error is now of lower-order since the term $C_{\Psi}^{n} \tau^{3 / 2}$ has been replaced by $t_{*}^{1 / 2} \tilde{C}_{\Psi}^{n} \tau$.

Lemma 4.13. Assume $\gamma=\gamma_{*}$ and the bound (4.29) on the Courant number. Assume the additional hyperbolictype restriction on the time step,

$$
\tau \leq t_{*}^{1 / 2} \mu^{-1 / 2} h
$$

Then,

$$
\frac{1}{2}\left\|\xi_{h}^{n+1}\right\|_{L}^{2}-\frac{1}{2}\left\|\xi_{h}^{n}\right\|_{L}^{2}+\frac{1}{8} \tau\left|\theta_{h}^{n}\right|_{S}^{2}+\frac{1}{8} \tau\left|\zeta_{h}^{n}\right|_{S}^{2}+\frac{1}{80} c_{a} \tau\left\|\xi_{h}^{n+1}\right\|_{A}^{2} \lesssim \tau\left(\tilde{E}_{h}^{n}\right)^{2} .
$$

Proof. We take the $L$-scalar product of (4.27) with $\tau A_{h} \xi_{h}^{n+1}$ to infer

$$
\tau\left(\xi_{h}^{n+1}, A_{h} \xi_{h}^{n+1}\right)_{L}=\tau\left(T_{1}+T_{2}, A_{h} \xi_{h}^{n+1}\right)_{L}+\tau^{2}\left(T_{3}-\Xi_{h}^{n}-\Psi_{h}^{n}, A_{h} \xi_{h}^{n+1}\right)_{L},
$$

where

$$
T_{1}=\left(1+\omega_{1}\right) \zeta_{h}^{n}+\left(\omega_{2}-\omega_{1}\right) \theta_{h}^{n}, \quad T_{2}=-\omega_{2} \xi_{h}^{n}, \quad T_{3}=-\frac{1}{2} B_{h}\left(\zeta_{h}^{n}-\theta_{h}^{n}\right)+\omega_{1} B_{h} \theta_{h}^{n} .
$$


Since $\gamma=\gamma_{*}, \omega_{2}=0$ so that $T_{2}=0$. We now bound the other terms on the right-hand side of (4.34). To bound the term with $T_{1}$, we use the boundedness (2.26) of $A_{h}$ to infer

$$
\tau\left(T_{1}, A_{h} \xi_{h}^{n+1}\right)_{L} \lesssim \tau\left(\left\|\theta_{h}^{n}\right\|_{A}+\left\|\zeta_{h}^{n}\right\|_{A}\right)\left\|\xi_{h}^{n+1}\right\|_{A} .
$$

To bound the term with $T_{3}$, we use the Cauchy-Schwarz inequality, (4.24), (2.26), and Co $\leq 1$, yielding

$$
\tau^{2}\left(B_{h} \theta_{h}^{n}, A_{h} \xi_{h}^{n+1}\right)_{L} \leq \tau^{2}\left\|B_{h} \theta_{h}^{n}\right\|_{L}\left\|A_{h} \xi_{h}^{n+1}\right\|_{L} \lesssim \tau^{2} \sigma \mu^{-1 / 2}\left\|\theta_{h}^{n}\right\|_{A} \mu^{1 / 2} h^{-1}\left\|\xi_{h}^{n+1}\right\|_{A} \leq \tau\left\|\theta_{h}^{n}\right\|_{A}\left\|\xi_{h}^{n+1}\right\|_{A} .
$$

Proceeding similarly for the contribution of $\zeta_{h}^{n}$, we infer

$$
\tau^{2}\left(T_{3}, A_{h} \xi_{h}^{n+1}\right)_{L} \lesssim \tau\left(\left\|\theta_{h}^{n}\right\|_{A}+\left\|\zeta_{h}^{n}\right\|_{A}\right)\left\|\xi_{h}^{n+1}\right\|_{A}
$$

To bound the term with $\Xi_{h}^{n}$, recalling (4.28), we first observe using (2.26) that

$$
\tau^{2}\left(B_{h} \theta_{\pi}^{n}, A_{h} \xi_{h}^{n+1}\right)_{L} \lesssim \tau^{2}\left\|B_{h} \theta_{\pi}^{n}\right\|_{A}\left\|\xi_{h}^{n+1}\right\|_{A} \lesssim \tau\left(\left\|\theta_{\pi}^{n}\right\|_{A *}+\left(\frac{\mu}{\sigma h}\right)^{1 / 2}\left|\theta_{\pi}^{n}\right|_{S}\right)\left\|\xi_{h}^{n+1}\right\|_{A},
$$

since owing to (2.26), (2.20), and $\mathrm{Co} \leq 1$,

$$
\begin{aligned}
\tau\left\|B_{h} \theta_{\pi}^{n}\right\|_{A} & \lesssim \tau \mu^{1 / 2} h^{-1}\left\|B_{h} \theta_{\pi}^{n}\right\|_{L} \lesssim \tau \mu^{1 / 2} h^{-1}\left(\sigma\left\|\nabla \theta_{\pi}^{n}\right\|_{L^{d}}+\sigma^{1 / 2} h^{-1 / 2}\left|\theta_{\pi}^{n}\right|_{S}\right) \\
& \leq \mu^{1 / 2}\left\|\nabla \theta_{\pi}^{n}\right\|_{L^{d}}+\left(\frac{\mu}{\sigma h}\right)^{1 / 2}\left|\theta_{\pi}^{n}\right|_{S} .
\end{aligned}
$$

Proceeding similarly for the contribution of $\zeta_{\pi}^{n}$, we infer

$$
\tau^{2}\left(\Xi_{h}^{n}, A_{h} \xi_{h}^{n+1}\right)_{L} \lesssim \tau\left(\left\|\theta_{\pi}^{n}\right\|_{A *}+\left\|\zeta_{\pi}^{n}\right\|_{A *}+\left(\frac{\mu}{\sigma h}\right)^{1 / 2}\left(\left|\theta_{\pi}^{n}\right|_{S}+\left|\zeta_{\pi}^{n}\right|_{S}\right)\right)\left\|\xi_{h}^{n+1}\right\|_{A} .
$$

Finally, to bound the term with $\Psi_{h}^{n}$, we use the Cauchy-Schwarz inequality and (2.26) to infer

$$
\tau^{2}\left(\Psi_{h}^{n}, A_{h} \xi_{h}^{n+1}\right)_{L} \leq \tau^{2}\left\|\Psi_{h}^{n}\right\|_{L} \mu^{1 / 2} h^{-1}\left\|\xi_{h}^{n+1}\right\|_{A} \leq \tau t_{*}^{1 / 2}\left\|\Psi_{h}^{n}\right\|_{L}\left\|\xi_{h}^{n+1}\right\|_{A} \leq \tau \tilde{E}_{h}^{n}\left\|\xi_{h}^{n+1}\right\|_{A}
$$

owing to the assumption (4.32) on the time step and the fact that $t_{*}^{1 / 2}\left\|\Psi_{h}^{n}\right\|_{L} \leq \tilde{E}_{h}^{n}$ owing to (3.15a). Combining the above bounds and using the discrete stability (2.17), we obtain

$$
\tau c_{a}\left\|\xi_{h}^{n+1}\right\|_{A}^{2} \lesssim \tau\left(\left\|\theta_{h}^{n}\right\|_{A}+\left\|\zeta_{h}^{n}\right\|_{A}+\tilde{E}_{h}^{n}\right)\left\|\xi_{h}^{n+1}\right\|_{A},
$$

whence the conclusion is straightforward using the stability estimate (4.30).

Remark 4.14 (restrictions on the time step). When the Péclet number is small, condition (4.29) simply reduces to $\mathrm{Co} \leq 1$. In the pure-diffusion limit, this condition, in turn, becomes trivial, and the only restriction on the time step is (4.32), which is needed to handle the truncation error in Lemma 4.13. Note also that the conditions Co $\leq 1$ and (4.32) can be regrouped into the condition $\tau \leq t_{*}^{1 / 2} \bar{\mu}^{-1 / 2} h$ with $\bar{\mu}$ defined in Section 2.1.

Remark 4.15 (choice of $\gamma$ ). The parameter $\gamma$ can slightly deviate from the value $\gamma_{*}$, but this leads to a more stringent bound on the Courant number than (4.29). Using (4.27), for positive real numbers $\epsilon$ and $\hat{\epsilon}$, we obtain

$$
\begin{aligned}
\frac{1}{2}\left\|\xi_{h}^{n+1}-\zeta_{h}^{n}\right\|_{L}^{2} & \leq \frac{1}{2}\left(1+\epsilon^{-1}\right) \tau^{2}\left\|X_{h}^{n}\right\|_{L}^{2}+\frac{1}{2}(1+\epsilon)\left\|\omega_{1}\left(\zeta_{h}^{n}-\theta_{h}^{n}\right)+\omega_{2}\left(\theta_{h}^{n}-\xi_{h}^{n}\right)\right\|_{L}^{2} \\
& \leq \frac{1}{2}\left(1+\epsilon^{-1}\right) \tau^{2}\left\|X_{h}^{n}\right\|_{L}^{2}+\frac{1}{2}(1+\epsilon)(1+\hat{\epsilon}) \omega_{1}^{2}\left\|\zeta_{h}^{n}-\theta_{h}^{n}\right\|_{L}^{2}+\frac{1}{2}(1+\epsilon)\left(1+\hat{\epsilon}^{-1}\right) \omega_{2}^{2}\left\|\theta_{h}^{n}-\xi_{h}^{n}\right\|_{L}^{2}
\end{aligned}
$$


For $\gamma \in\left[\frac{1}{4}, \frac{1}{2}\right], \omega_{1}$ is a decreasing function of $\gamma$ taking values in $[0,1]$, while $\omega_{2}$ is an increasing function of $\gamma$ taking values in $[-1,1]$ with $\omega_{2}\left(\gamma_{*}\right)=0$. The proof of Lemma 4.12 can be extended as long as there is $\hat{\epsilon}>0$ such that $\frac{1}{2}(1+\hat{\epsilon}) \omega_{1}^{2} \leq \frac{3}{8}$ and $\frac{1}{2}\left(1+\hat{\epsilon}^{-1}\right) \omega_{2}^{2} \leq \frac{1}{2}$ exploiting the presence of the term $\frac{1}{2}\left\|\theta_{h}^{n}-\zeta_{h}^{n}\right\|_{L}^{2}$ on the left-hand side of (4.25). A direct verification shows that this is possible as long as $\gamma \in\left(\gamma_{* *}, \frac{1}{2}\right)$ with $\gamma_{* *}=(2+\sqrt{8 / 3})^{-1} \simeq 0.275$ (corresponding to $\omega_{1}=\sqrt{2 / 3}$ and $\omega_{2}=-1 / 3$ ). We observe that the above numerical values depend on the choice $\alpha=\frac{3}{4}$ made in the proof of Lemma 4.10. Taking a larger value for $\alpha<1$ yields a more stringent bound on the Courant number in Lemma 4.10 but more flexibility in the choice of $\gamma$. Finally, the result of Lemma 4.13 is slightly modified since bounding the term $\omega_{2} \tau\left(\xi_{h}^{n}, A_{h} \xi_{h}^{n+1}\right)_{L}$ by Young's inequality leads to an additional term on the right-hand of (4.33) of the form $\frac{1}{80} \lambda c_{a} \tau\left\|\xi_{h}^{n}\right\|_{A}^{2}$ where $\lambda$ can be chosen $<1$ provided $\gamma$ is sufficiently close to $\gamma_{*}$ so that $\omega_{2}$ is sufficiently small. Details are skipped for brevity.

We can now derive our main convergence result in the diffusion-dominated regime.

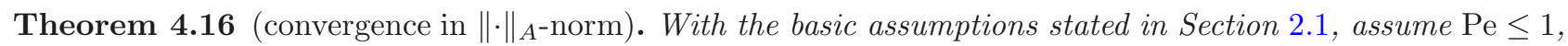
take $\gamma=\gamma_{*}$, and assume the bound (4.29) on the Courant number together with the bound (4.32) on the time step. Then,

$$
\left(\tau \sum_{n=1}^{N}\left\|u^{n}-u_{h}^{n}\right\|_{A}^{2}\right)^{1 / 2} \lesssim \tilde{C}_{\mathrm{tim}} t_{*}^{1 / 2} \tau+\tilde{C}_{\mathrm{spc}} \mu^{1 / 2} h
$$

where $\tilde{C}_{\mathrm{tim}}^{2}=\sum_{n=0}^{N-1} \tau\left(\tilde{C}_{\Psi}^{n}\right)^{2}$ and $\tilde{C}_{\mathrm{spc}}^{2}=\sum_{n=0}^{N-1} \tau\left(\left(\tilde{K}_{2}^{n}\right)^{2}+(\tau / \mu)\left(K_{w-u}^{n}\right)^{2}\right)$.

Proof. Using the stability result of Lemma 4.13 , we sum over $n$, discard the $|\cdot|_{S}$-terms on the left-hand side, and use a discrete Gronwall lemma to eliminate the contribution of $\left\|\xi_{h}^{n}\right\|_{L}^{2}$ in $\tilde{E}_{h}^{n}$. This yields

$$
\tau \sum_{n=1}^{N}\left\|\xi_{h}^{n}\right\|_{A}^{2} \lesssim \sum_{n=0}^{N-1} \tau\left(\left\|\theta_{\pi}^{n}\right\|_{B *}^{2}+\left\|\zeta_{\pi}^{n}\right\|_{B *}^{2}+\left\|\theta_{\pi}^{n}\right\|_{A *}^{2}+\left\|\zeta_{\pi}^{n}\right\|_{A *}^{2}+\operatorname{Pe}^{-1}\left(\left|\theta_{\pi}^{n}\right|_{S}^{2}+\left|\zeta_{\pi}^{n}\right|_{S}^{2}\right)+t_{*}\left(\tilde{C}_{\Psi}^{n}\right)^{2} \tau^{2}\right)
$$

To bound the terms with $\theta_{\pi}^{n}$ and $\zeta_{\pi}^{n}$, we use the result of Lemma 3.8 for the $\|\cdot\|_{A^{-}}$and $\|\cdot\|_{B *^{-}}$norm, while for the $|\cdot|_{S}$-seminorm, we use the bounds (3.6) and (3.8) on $\left|v^{n}\right|_{H^{2}}$ and $\left|w^{n}\right|_{H^{2}}$ and $\left|u^{n}\right|_{H^{2}} \leq \tilde{K}_{2}^{n}$ to infer

$$
\mathrm{Pe}^{-1 / 2}\left(\left|\theta_{\pi}^{n}\right|_{S}+\left|\zeta_{\pi}^{n}\right|_{S}\right) \lesssim \mu^{1 / 2} h\left(\left|v^{n}\right|_{H^{2}}+\left|w^{n}\right|_{H^{2}}\right) \lesssim \mu^{1 / 2} h \tilde{K}_{2}^{n}+\tau^{1 / 2} h K_{w-u}^{n}
$$

The conclusion is straightforward using $\sigma^{1 / 2} h^{1 / 2} \leq \mu^{1 / 2}$ since $\mathrm{Pe} \leq 1$.

It is possible to derive an $L$-norm error estimate with higher convergence rates than (4.35). The proof is postponed to Section 7.2.

Proposition 4.17 (convergence in $L$-norm). Assume that $\beta$ has bounded second-order derivatives with associated bound denoted by $\sigma_{2}$. Then, under the assumptions of Theorem 4.16, there holds

$$
\left\|u^{N}-u_{h}^{N}\right\|_{L} \lesssim C_{\mathrm{tim}} \tau^{3 / 2}+\hat{C}_{\mathrm{spc}} \sigma^{1 / 2} h^{3 / 2}+\hat{C}_{\mathrm{spc}}^{\prime} \mu^{-1 / 2} h^{2}
$$

where $C_{\mathrm{tim}}$ is defined in Theorem 4.8, $\left(\hat{C}_{\mathrm{spc}}\right)^{2}=\sum_{n=0}^{N-1} \tau\left(\hat{K}_{w-u}^{n}\right)^{2},\left(\hat{C}_{\mathrm{spc}}^{\prime}\right)^{2}=\sum_{n=0}^{N-1} \tau C_{P}^{2}\left(K_{2}^{n}+\left\|\partial_{t} u\right\|_{C\left(I_{n} ; H^{2}\right)}\right)^{2}$, with $\hat{K}_{w-u}^{n}=C_{P}\left(\left|u^{n}\right|_{H^{3}}+(\tau / \mu)^{1 / 2} K_{2}^{n}+\sigma^{-1}\left(\sigma_{1} \tilde{K}_{2}^{n}+\sigma_{2} \tilde{K}_{1}^{n}\right)\right)+\tilde{K}_{2}^{n}+(\tau / \mu)^{1 / 2} K_{w-u}^{n}$ and $C_{P}$ is the global length scale associated with the Poincaré inequality stating that for all $v_{h} \in V_{h},\left\|v_{h}\right\|_{L} \leq \mu^{-1 / 2} C_{P}\left\|v_{h}\right\|_{A}$. 


\section{ExTENSIONS}

For simplicity, the above analysis was presented in the case where space discretization was performed using continuous, piecewise affine finite elements with CIP. Other finite element methods with symmetric stabilization can be used. This requires establishing discrete stability and boundedness for the discrete operators $B_{h}$ and $A_{h}$. For consistent methods, the stability and convergence analysis of Section 4 can then be readily applied, while minor adaptations are needed in the case of weakly consistent methods to formulate the truncation errors.

To illustrate, we briefly consider a DG method for space discretization using upwinding for the advective part and symmetric interior penalty for the diffusive part. Let $V_{h}^{\text {d }}$ denote the space spanned by (discontinuous) piecewise affine functions on the mesh $\mathcal{T}_{h}$. For a smooth enough function $v$ that is possibly double-valued at $F \in \mathcal{F}_{h}^{\text {int }}$ with $F=\partial T^{-} \cap \partial T^{+}$, we define, in addition to its jump, its mean value as $\left\{\{v\}:=\frac{1}{2}\left(\left.v\right|_{T^{-}}+\left.v\right|_{T^{+}}\right)\right.$. On boundary faces, the jump and mean value refer to the actual value of $v$ on $F$. The discrete operators $B_{h}$ and $A_{h}$ are now such that

$$
\begin{aligned}
\left(B_{h} z, w_{h}\right)_{L}:= & \left(\beta \cdot \nabla_{h} z, w_{h}\right)_{L}-\sum_{F \in \mathcal{F}_{h}^{\text {int }}}\left(\left(\nu_{F} \cdot \beta\right) \llbracket z \rrbracket,\left\{\left\{w_{h}\right\}\right)_{L, F}+\sum_{F \in \mathcal{F}_{h}^{\text {int }}} S_{\text {upw }}\left(\left|\nu_{F} \cdot \beta\right| \llbracket z \rrbracket, \llbracket w_{h} \rrbracket\right)_{L, F},\right. \\
\left(A_{h} z, w_{h}\right)_{L}= & \mu\left(\nabla_{h} z, \nabla_{h} w_{h}\right)_{L^{d}}-\sum_{F \in \mathcal{F}_{h}} \mu\left(\nu_{F} \cdot\left\{\left\{\nabla_{h} z\right\}\right\}, \llbracket w_{h} \rrbracket\right)_{L, F}-\sum_{F \in \mathcal{F}_{h}} \mu\left(\llbracket z \rrbracket, \nu_{F} \cdot\left\{\left\{\nabla_{h} w_{h}\right\}\right\}\right)_{L, F} \\
& +\sum_{F \in \mathcal{F}_{h}} S_{\mathrm{ip}} \mu h_{F}^{-1}\left(\llbracket z \rrbracket, \llbracket w_{h} \rrbracket\right)_{L, F},
\end{aligned}
$$

where $\nabla_{h}$ denotes the broken (elementwise) gradient operator, while $S_{\text {upw }}=\frac{1}{2}$ for classical upwinding, and $S_{\text {ip }}$ is taken large enough. Then, letting

$$
|z|_{S}^{2}:=\sum_{F \in \mathcal{F}_{h}^{\text {int }}} \frac{1}{2}\left\|\left|\nu_{F} \cdot \beta\right|^{1 / 2} \llbracket z \rrbracket\right\|_{L, F}^{2}, \quad\|z\|_{A}^{2}:=\mu\|\nabla z\|_{L^{d}}^{2}+\sum_{F \in \mathcal{F}_{h}} \mu h_{F}^{-1}\|\llbracket z \rrbracket\|_{L, F}^{2},
$$

it is readily verified that the discrete stability properties stated in Section 2.6 hold true. Moreover, letting

$$
\begin{aligned}
& \|z\|_{B *}:=|z|_{S}+\sigma^{1 / 2} h^{-1 / 2}\|z\|_{L}+\left(\sum_{T \in \mathcal{T}_{h}} \sum_{F \subset \partial T} \sigma\|z\|_{L, F}^{2}\right)^{1 / 2}, \\
& \|z\|_{A *}:=\|z\|_{A}+\left(\sum_{T \in \mathcal{T}_{h}} \sum_{F \subset \partial T} \mu h_{F}\left\|\nu_{F} \cdot \nabla z\right\|_{L, F}^{2}\right)^{1 / 2},
\end{aligned}
$$

it is readily verified that the boundedness properties stated in Section 2.6 hold true.

It is also possible to consider higher-order continuous or discontinuous finite elements with symmetric stabilization. To achieve stability, the sole modification in the above analysis concerns the advection-dominated regime, since the boundedness property (2.22) can no longer be used. It is then necessary to modify the proof of Lemma 4.5 when bounding $\frac{1}{2}\left\|\xi_{h}^{n+1}-\zeta_{h}^{n}\right\|_{L}^{2}$. In particular, following [8] (details are skipped for brevity), the term $\frac{1}{2} \tau B_{h} \eta_{h}^{n}$ on the right-hand side of (4.19) is controlled by the so-called 4/3-CFL condition $\tau \lesssim t_{*}^{-1 / 3}(h / \sigma)^{4 / 3}$. Deriving convergence rates is a more delicate question not covered herein; it entails, in particular, obtaining bounds for higher-order Sobolev norms of the auxiliary functions $v^{n}$ and $w^{n}$.

\section{NumERICAL EXAMPLES}

We consider two numerical experiments using FreeFem $++[22]$ to illustrate the above analysis, namely convergence rates to a known smooth solution and control of spurious oscillations for a solution with sharp layers. For all flow regimes, we used $\gamma=1-\frac{1}{\sqrt{2}}$ and set the penalty parameters to $S_{\text {cip }}=0.005$ and $S_{\mathrm{bc}}=10$ for CIP and to $S_{\mathrm{ip}}=10$ for DG. 
TABLE 1. Convergence for smooth solution.

\begin{tabular}{|c|cc|cc|c|c|}
\hline$m$ & \multicolumn{4}{|c|}{$\mu=0.1$} & \multicolumn{2}{c|}{$\mu=10^{-4}$} \\
\hline & \multicolumn{2}{|c|}{ CIP } & \multicolumn{2}{c|}{ DG } & CIP & DG \\
& Final $L^{2}$ & $L^{2}\left(H^{1}\right)$ & Final $L^{2}$ & $L^{2}\left(H^{1}\right)$ & Final $L^{2}$ & Final $L^{2}$ \\
\hline 0 & $2.8 \mathrm{e}-3$ & $5.7 \mathrm{e}-2$ & $2.0 \mathrm{e}-3$ & $7.2 \mathrm{e}-2$ & - & - \\
1 & $9.5 \mathrm{e}-4$ & $2.7 \mathrm{e}-2$ & $7.8 \mathrm{e}-3$ & $4.0 \mathrm{e}-2$ & $4.6 \mathrm{e}-2$ & $1.6 \mathrm{e}-2$ \\
2 & $2.2 \mathrm{e}-4$ & $1.0 \mathrm{e}-2$ & $1.7 \mathrm{e}-4$ & $1.8 \mathrm{e}-2$ & $1.0 \mathrm{e}-2$ & $3.2 \mathrm{e}-3$ \\
3 & $5.3 \mathrm{e}-5$ & $5.5 \mathrm{e}-3$ & $4.2 \mathrm{e}-5$ & $9.1 \mathrm{e}-3$ & $1.4 \mathrm{e}-3$ & $5.2 \mathrm{e}-4$ \\
4 & - & - & - & - & $2.1 \mathrm{e}-4$ & $9.2 \mathrm{e}-5$ \\
\hline
\end{tabular}

\subsection{Convergence to smooth solutions}

Let $\Omega=\left\{r^{2}:=x^{2}+y^{2}<2\right\}$ and consider the rotating velocity field $\beta=(y,-x)^{T}$ so that $\sigma=2$. Letting $\mathbf{x}=(x, y)^{T}$, the exact solution is chosen to be the advected heat kernel in the form

$$
u(\mathbf{x}, t)=\frac{\ell_{0}^{2}}{t \mu+\ell_{0}^{2}} \exp \left(-\frac{|\mathbf{r}(t)-\mathbf{x}|^{2}}{4\left(\mu t+\ell_{0}^{2}\right)}\right), \quad \mathbf{r}(t)=(0.3 \sin (t), 0.3 \cos (t))^{T},
$$

where the length scale $\ell_{0}=0.1$ determines the spread of the initial Gaussian. We consider two settings, first $\mu=0.1$ and $t_{\mathrm{F}}=\pi / 4$ and then $\mu=10^{-4}$ and $t_{\mathrm{F}}=2 \pi$. In both cases, the decay of the exact solution away from $\mathbf{r}(t)$ is sufficiently fast to enforce homogeneous Dirichlet conditions on $\partial \Omega$. We discretize the boundary $\partial \Omega$ with $M$ elements from which a quasi-uniform triangulation of $\Omega$ is constructed, yielding a mesh size $h=4 \pi / M$. We take $M=2^{6+m}$ with $m \in\{0,1,2,3,4\}$. For $\mu=0.1$, the Péclet number decays from 4 to 0.25 corresponding to a diffusion-dominated regime, while for $\mu=10^{-4}$, the Péclet number is $10^{3}$ times larger, corresponding to an advection-dominated regime. In both regimes, the time step is selected by setting the Courant number to $\mathrm{Co}=\frac{1}{2}$ for CIP, and using the tighter restriction $\mathrm{Co}=\frac{1}{8}$ for DG in the advection-dominated regime. Results are reported in Table 1 for the $L^{2}$-error at final time and the $L^{2}\left(0 ; t_{\mathrm{F}} ; H_{0}^{1}(\Omega)\right)$-error. For $\mu=0.1$, the result on the finest mesh is omitted since the mesh is sufficiently fine, and the diffusion coefficient sufficiently large, to detect the influence of using homogeneous Dirichlet boundary conditions; for $\mu=10^{-4}$, the result on the coarsest mesh is omitted since the mesh is too coarse to resolve the initial datum. In all cases, the convergence rates match, or are slightly better than, those predicted by the theory.

\subsection{Solutions with sharp layers}

The purpose of this test case is to illustrate numerically that in the advection-dominated regime, spurious oscillations resulting from insufficient mesh resolution of sharp layers do not spread over the whole domain, but remain contained at all times close to the layer. Let $\mu=10^{-6}$ and consider the initial datum

$$
u_{0}(\mathbf{x})=0.5\left(\tanh \left(\left(\exp \left(-20\left|\mathbf{r}\left(\frac{\pi}{4}\right)-\mathbf{x}\right|^{2}\right)-0.5\right) / 0.0001\right)+1\right) .
$$

The graph of $u_{0}$ corresponds to a cylinder centered at $\mathbf{x}=(0.3,0.3)^{T}$. The width of the inner layer is $10^{-4}$. The mesh is built using $M=512$ so that it does not resolve this inner layer. The final time is $t_{\mathrm{F}}=2 \pi$ corresponding to one full rotation of the initial datum. Figure 1 displays the initial datum, the CIP approximate solution without stabilization $\left(S_{\text {cip }}=0\right)$, and the solution with stabilization $\left(S_{\text {cip }}=0.005\right)$. The unstabilized solution exhibits global spurious oscillations, while the improved quality of the stabilized solution is clearly visible. Finally, Table 2 reports $L^{2}$-errors at final time obtained using CIP and DG (with $\mu=0$ so that the analytical solution is known explicitly). Both space approximation methods exhibit the same convergence behavior. The convergence rate is of order $h^{1 / 4}$ as often observed for rough solutions on general meshes. 
TABLE 2. Convergence for rough solution; $L^{2}$-error at final time.

\begin{tabular}{|l|c|c|}
\hline$m$ & CIP & DG \\
\hline 0 & $1.9 \mathrm{e}-1$ & $1.2 \mathrm{e}-1$ \\
1 & $1.4 \mathrm{e}-1$ & $1.0 \mathrm{e}-1$ \\
2 & $1.1 \mathrm{e}-1$ & $8.2 \mathrm{e}-2$ \\
3 & $8.8 \mathrm{e}-2$ & $6.9 \mathrm{e}-2$ \\
4 & $7.0 \mathrm{e}-2$ & $5.6 \mathrm{e}-2$ \\
\hline
\end{tabular}
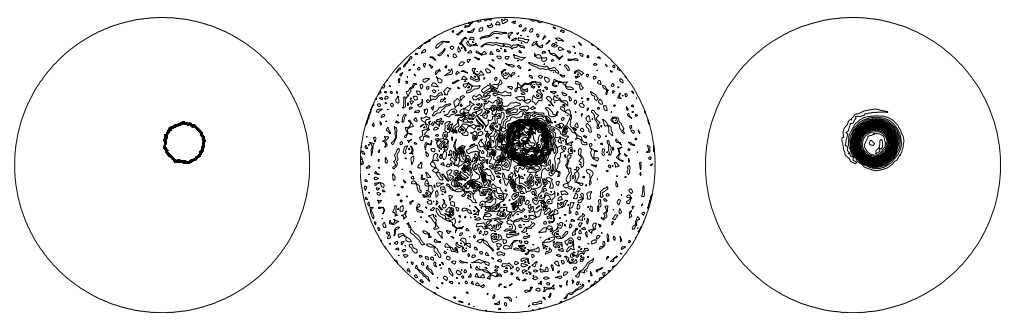

Figure 1. Initial datum (left) and solution at final time without (middle) and with (right) CIP stabilization.

\section{Proofs of Propositions 4.9 And 4.17}

This sections collects the proofs of Propositions 4.9 and 4.17 .

\subsection{Proof of Proposition 4.9}

The proof, which proceeds along that of Lemma 4.13, is only sketched. There are essentially two differences. Firstly, the term $T_{2}$ in this proof needs to be bounded since we do not assume here that $\gamma=\gamma_{*}$. To this purpose, we use (2.15a) and the definition of $\alpha_{h}^{n}$ to obtain

$$
\tau\left(\xi_{h}^{n}, A_{h} \xi_{h}^{n+1}\right)_{L}=\tau\left(\theta_{h}^{n}, A_{h} \xi_{h}^{n+1}\right)_{L}+\gamma \tau^{2}\left(A_{h} \theta_{h}^{n}, A_{h} \xi_{h}^{n+1}\right)_{L}-\gamma \tau^{2}\left(A_{h} \theta_{\pi}^{n}, A_{h} \xi_{h}^{n+1}\right)_{L} .
$$

The first term on the right-hand side is treated as the term $T_{1}$ in the proof of Lemma 4.13. For the second term, the Cauchy-Schwarz inequality and (4.15) yield

$$
\tau^{2}\left(A_{h} \theta_{h}^{n}, A_{h} \xi_{h}^{n+1}\right)_{L} \leq \tau^{2}\left\|A_{h} \theta_{h}^{n}\right\|_{L}\left\|A_{h} \xi_{h}^{n+1}\right\|_{L} \lesssim(\mathrm{Co} / \mathrm{Pe}) \tau\left\|\theta_{h}^{n}\right\|_{A}\left\|\xi_{h}^{n+1}\right\|_{A} \leq \tau\left\|\theta_{h}^{n}\right\|_{A}\left\|\xi_{h}^{n+1}\right\|_{A},
$$

since $\mathrm{Co} \leq 1$ and $\mathrm{Pe} \geq 1$. Finally, for the third term, the Cauchy-Schwarz inequality, (4.15), and (2.25) lead to

$$
\tau^{2}\left(A_{h} \theta_{\pi}^{n}, A_{h} \xi_{h}^{n+1}\right)_{L} \leq \tau^{2}\left\|A_{h} \theta_{\pi}^{n}\right\|_{L}\left\|A_{h} \xi_{h}^{n+1}\right\|_{L} \lesssim \tau\left\|\theta_{\pi}^{n}\right\|_{A *}\left\|\xi_{h}^{n+1}\right\|_{A}
$$

since $\tau^{1 / 2} \mu^{1 / 2} h^{-1}=(\mathrm{Co} / \mathrm{Pe})^{1 / 2} \leq 1$. Collecting these estimates, we infer

$$
\tau\left(T_{2}, A_{h} \xi_{h}^{n+1}\right)_{L} \lesssim \tau\left(\left\|\theta_{h}^{n}\right\|_{A}+\left\|\theta_{\pi}^{n}\right\|_{A *}\right)\left\|\xi_{h}^{n+1}\right\|_{A} .
$$

Secondly, when dealing with the truncation error in time, we exploit the fact that $\mathrm{Pe} \geq 1$ to derive a sharper bound than in the proof of Lemma 4.13, namely

$$
\tau^{2}\left(\Psi_{h}^{n}, A_{h} \xi_{h}^{n+1}\right)_{L} \leq \tau^{2}\left\|\Psi_{h}^{n}\right\|_{L} \mu^{1 / 2} h^{-1}\left\|\xi_{h}^{n+1}\right\|_{A} \leq(\mathrm{Co} / \mathrm{Pe})^{1 / 2} \tau^{3 / 2}\left\|\Psi^{n}\right\|_{L}\left\|\xi_{h}^{n+1}\right\|_{A} \lesssim \tau E_{h}^{n}\left\|\xi_{h}^{n+1}\right\|_{A},
$$

where we have used (4.21). As a result, an estimate similar to (4.33) is inferred, but with a quantity $\hat{E}_{h}^{n}$ on the right-hand side which is defined as (4.31) except that $t_{*}^{1 / 2} \tilde{C}_{\Psi}^{n} \tau$ is replaced by the sharper estimate $C_{\Psi}^{n} \tau^{3 / 2}$. The conclusion is straightforward using, in particular, that

$$
\mathrm{Pe}^{-1 / 2}\left(\left|\theta_{\pi}^{n}\right|_{S}+\left|\zeta_{\pi}^{n}\right|_{S}\right) \lesssim \mu^{1 / 2} h\left(\left|v^{n}\right|_{H^{2}}+\left|w^{n}\right|_{H^{2}}\right) \lesssim \mu^{1 / 2} h \tilde{K}_{2}^{n}+\tau^{1 / 2} h K_{w-u}^{n} \leq \sigma^{1 / 2} h^{3 / 2}\left(\tilde{K}_{2}^{n}+\sigma^{-1} K_{w-u}^{n}\right),
$$

since $\mathrm{Pe} \geq 1$ and $\mathrm{Co} \leq 1$. 


\subsection{Proof of Proposition 4.17}

For brevity, we only sketch the proof. We introduce the discrete Riesz projection of $u^{n}$ and of the auxiliary functions $v^{n}$ and $w^{n}$. Specifically, $r_{h} u^{n} \in V_{h}$ is defined such that $A_{h} r_{h} u^{n}:=A_{h} u^{n}$ and similarly for $r_{h} v^{n}$ and $r_{h} w^{n}$. Then, redefining the quantities $\xi_{h}^{n}:=u_{h}^{n}-r_{h} u^{n}, \xi_{\pi}^{n}:=u^{n}-r_{h} u^{n}$ and similarly for $\theta_{h}^{n}, \theta_{\pi}^{n}, \zeta_{h}^{n}$, and $\zeta_{\pi}^{n}$, the error equation takes again the form (2.15) with the new source terms

$$
\begin{aligned}
\alpha_{h}^{n} & =\tau^{-1} \pi_{h}\left(I-r_{h}\right)\left(v^{n}-u^{n}\right), \quad \beta_{h}^{n}=\tau^{-1} \pi_{h}\left(I-r_{h}\right)\left(w^{n}-v^{n}\right)-B_{h}\left(I-r_{h}\right) v^{n}, \\
\delta_{h}^{n} & =\tau^{-1} \pi_{h}\left(I-r_{h}\right)\left(u^{n+1}-\frac{1}{2}\left(v^{n}+w^{n}\right)\right)-\frac{1}{2} B_{h}\left(I-r_{h}\right) w^{n} .
\end{aligned}
$$

Then, the basic energy identity of Lemma 4.1 is not modified. Instead, the basic energy estimate of Theorem 4.4 requires bounding the new source terms. Using the Cauchy-Schwarz inequality, the Poincaré inequality, the approximation properties of the Riesz projector $r_{h}$, the bound (3.5) on $\left\|\Delta\left(v^{n}-u^{n}\right)\right\|_{L}$, and elliptic regularity, we obtain

$$
\tau\left(\alpha_{h}^{n}, \theta_{h}^{n}\right)_{L} \leq \mu^{-1 / 2} C_{P}\left\|\left(I-r_{h}\right)\left(v^{n}-u^{n}\right)\right\|_{L}\left\|\theta_{h}^{n}\right\|_{A} \lesssim \mu^{-1 / 2} C_{P} h^{2}\left|v^{n}-u^{n}\right|_{H^{2}}\left\|\theta_{h}^{n}\right\|_{A} \lesssim \mu^{-1 / 2} C_{P} h^{2} \tau K_{2}^{n}\left\|\theta_{h}^{n}\right\|_{A} .
$$

Hence, by Young's inequality,

$$
\tau\left(\alpha_{h}^{n}, \theta_{h}^{n}\right)_{L} \leq C \tau\left(\mu^{-1 / 2} C_{P} h^{2} K_{2}^{n}\right)^{2}+\lambda \tau\left\|\theta_{h}^{n}\right\|_{A}^{2},
$$

where $\lambda$ can be chosen as small as needed. To bound $\tau\left(\beta_{h}^{n}, \theta_{h}^{n}\right)_{L}$, we write $w^{n}-v^{n}=\left(w^{n}-u^{n}\right)-\left(v^{n}-u^{n}\right)$, and estimate the contribution of $\left(v^{n}-u^{n}\right)$ as for $\alpha_{h}^{n}$. To bound the contribution of $\left(w^{n}-u^{n}\right)$, we observe that

$$
\left\|\left(I-r_{h}\right)\left(w^{n}-u^{n}\right)\right\|_{L} \lesssim h^{2}\left|w^{n}-u^{n}\right|_{H^{2}} \lesssim h^{2}\left\|\Delta\left(w^{n}-u^{n}\right)\right\|_{L} .
$$

We use a different bound on $\left\|\Delta\left(w^{n}-u^{n}\right)\right\|_{L}$ than (3.7), whereby we exploit that the advection field $\beta$ has bounded second-order derivatives. Letting $\mathfrak{v}$ denote the right-hand side of (3.9) and observing that $\mathfrak{v} \in V,(3.4)$ yields $\left\|\Delta\left(w^{n}-u^{n}\right)\right\|_{L} \lesssim\|\Delta \mathfrak{v}\|_{L}$. Using the bounds (3.6) on $v^{n}$ and the bound (3.5) on $\left\|\nabla \Delta\left(v^{n}-u^{n}\right)\right\|_{L^{d}}$, we infer $\left\|\Delta\left(B v^{n}\right)\right\|_{L} \lesssim \sigma \tilde{K}_{w-u}^{n}$ with $\tilde{K}_{w-u}^{n}=\left|u^{n}\right|_{H^{3}}+(\tau / \mu)^{1 / 2} K_{2}^{n}+\sigma^{-1}\left(\sigma_{1} \tilde{K}_{2}^{n}+\sigma_{2} \tilde{K}_{1}^{n}\right)$. Hence, $\left\|\Delta\left(w^{n}-u^{n}\right)\right\|_{L} \lesssim$ $\tau\left(K_{2}^{n}+\sigma \tilde{K}_{w-u}^{n}\right)$, so that

$$
\left\|\left(I-r_{h}\right)\left(w^{n}-u^{n}\right)\right\|_{L} \lesssim \tau h^{2}\left(K_{2}^{n}+\sigma \tilde{K}_{w-u}^{n}\right) .
$$

Finally, for the last term in $\beta_{h}^{n}$, we obtain by integrating by parts the advective derivative that

$$
\left(B_{h}\left(I-r_{h}\right) v^{n}, \theta_{h}^{n}\right)_{L} \lesssim h^{2}\left|v^{n}\right|_{H^{2}} \sigma \mu^{-1 / 2}\left\|\theta_{h}^{n}\right\|_{A} \lesssim \sigma \mu^{-1 / 2} h^{2} \tilde{K}_{2}^{n}\left\|\theta_{h}^{n}\right\|_{A},
$$

since $\left|v^{n}\right|_{H^{2}} \lesssim \tilde{K}_{2}^{n}$. Collecting these bounds and introducing the Péclet number yields

$$
\tau\left(\beta_{h}^{n}, \theta_{h}^{n}\right)_{L} \leq C \tau\left(\mu^{-1 / 2} h^{2} C_{P} K_{2}^{n}+\operatorname{Pe}^{1 / 2} \sigma^{1 / 2} h^{3 / 2}\left(C_{P} \tilde{K}_{w-u}^{n}+\tilde{K}_{2}^{n}\right)\right)^{2}+\lambda \tau\left\|\theta_{h}^{n}\right\|_{A}^{2},
$$

where $\lambda$ can be chosen as small as needed. The bound on $\tau\left(\delta_{h}^{n}, \zeta_{h}^{n}\right)_{L}$ is obtained using similar arguments, in particular that $u^{n+1}-\frac{1}{2}\left(v^{n}+w^{n}\right)=\left(u^{n+1}-u^{n}\right)-\left(\frac{1}{2}\left(v^{n}+w^{n}\right)-u^{n}\right),\left\|\left(I-r_{h}\right)\left(u^{n+1}-u^{n}\right)\right\|_{L} \lesssim \tau h^{2}\left\|\partial_{t} u\right\|_{C\left(I_{n} ; H^{2}\right)}$, and that $\left|w^{n}\right|_{H^{2}} \lesssim \tilde{K}_{2}^{n}+(\tau / \mu)^{1 / 2} K_{w-u}^{n}$ owing to (3.8). Therefore, we recover the stability estimates (4.14) and (4.25) with

$$
E_{h}^{n}=\mu^{-1 / 2} h^{2} C_{P}\left(K_{2}^{n}+\left\|\partial_{t} u\right\|_{C\left(I_{n} ; H^{2}\right)}\right)+\operatorname{Pe}^{1 / 2} \sigma^{1 / 2} h^{3 / 2} \hat{K}_{w-u}^{n},
$$

with $\hat{K}_{w-u}^{n}=C_{P} \tilde{K}_{w-u}^{n}+\tilde{K}_{2}^{n}+(\tau / \mu)^{1 / 2} K_{w-u}^{n}$. The next step is the result of Lemma 4.11 where the identity (4.27) holds true with $\Xi_{h}^{n}=\omega_{2} \alpha_{h}^{n}+\left(\omega_{1}+\frac{1}{2}\right) \beta_{h}^{n}-\delta_{h}^{n}$. Then, proceeding as in Lemma 4.12, we need to control $\tau\left\|\Xi_{h}^{n}\right\|_{L}$. We observe that

$$
\tau\left\|B_{h}\left(I-r_{h}\right) v^{n}\right\|_{L}+\tau\left\|B_{h}\left(I-r_{h}\right) w^{n}\right\|_{L} \lesssim \tau \sigma h\left(\left|v^{n}\right|_{H^{2}}+\left|w^{n}\right|_{H^{2}}\right) \lesssim \tau^{1 / 2} \sigma^{1 / 2} h^{3 / 2}\left(\tilde{K}_{2}^{n}+(\tau / \mu)^{1 / 2} K_{w-u}^{n}\right) .
$$


Defining $\hat{E}_{h}^{n}$ as $E_{h}^{n}$ in (7.1) by dropping the $\mathrm{Pe}^{1 / 2}$ factor in the last term, that is,

$$
\hat{E}_{h}^{n}=\mu^{-1 / 2} h^{2} C_{P}\left(K_{2}^{n}+\left\|\partial_{t} u\right\|_{C\left(I_{n} ; H^{2}\right)}\right)+\sigma^{1 / 2} h^{3 / 2} \hat{K}_{w-u}^{n},
$$

we eventually infer $\tau\left\|\Xi_{h}^{n}\right\|_{L} \lesssim \tau^{1 / 2} \hat{E}_{h}^{n}$. Finally, accounting for the truncation error in time, we recover the stability estimate (4.30) with the right-hand side

$$
\bar{E}_{h}^{n}:=\mu^{-1 / 2} h^{2} C_{P}\left(K_{2}^{n}+\left\|\partial_{t} u\right\|_{C\left(I_{n} ; H^{2}\right)}\right)+\sigma^{1 / 2} h^{3 / 2} \hat{K}_{w-u}^{n}+C_{\Psi}^{n} \tau^{3 / 2},
$$

whence the conclusion is straightforward.

Remark 7.1 (optimality in $h$ ). We observe that the error term defined by (7.1) exhibits second-order convergence as $h \rightarrow 0$ owing to the presence of the $\mathrm{Pe}^{1 / 2}$ factor in the last term. This is no longer the case for the error term $\bar{E}_{h}^{n}$, where the loss of the $\mathrm{Pe}^{1 / 2}$ factor is caused by the contribution of $B_{h}$ when bounding the anti-dissipative term. Optimality is recovered for vanishing advection.

\section{REFERENCES}

[1] U.M. Ascher, S.J. Ruuth and R.J. Spiteri, Implicit-explicit Runge-Kutta methods for time-dependent partial differential equations. Special issue on time integration (Amsterdam, 1996). Appl. Numer. Math. 25 (1997) 151-167.

[2] U.M. Ascher, S.J. Ruuth and B.T.R. Wetton, Implicit-explicit methods for time-dependent partial differential equations. SIAM J. Numer. Anal. 32 (1995) 797-823.

[3] M. Braack, E. Burman, V. John and G. Lube, Stabilized finite element methods for the generalized Oseen problem. Comput. Methods Appl. Mech. Engrg. 196 (2007) 853-866.

[4] A.N. Brooks and T.J.R. Hughes, Streamline upwind/Petrov-Galerkin formulations for convection dominated flows with particular emphasis on the incompressible Navier-Stokes equations. FENOMECH'81, Part I, Stuttgart (1981). Comput. Methods Appl. Mech. Engrg. 32 (1982) 199-259.

[5] E. Burman, A unified analysis for conforming and nonconforming stabilized finite element methods using interior penalty. SIAM J. Numer. Anal. 43 (2005) 2012-2033 (electronic).

[6] E. Burman, Consistent SUPG-method for transient transport problems: Stability and convergence. Comput. Methods Appl. Mech. Engrg. 199 (2010) 1114-1123.

[7] E. Burman and A. Ern, A continuous finite element method with face penalty to approximate Friedrichs' systems. ESAIM: M2AN 41 (2007) 55-76.

[8] E. Burman, A. Ern and M.A. Fernández, Explicit Runge-Kutta schemes and finite elements with symmetric stabilization for first-order linear PDE systems. SIAM J. Numer. Anal. 48 (2010) 2019-2042.

[9] E. Burman and M.A. Fernández, Finite element methods with symmetric stabilization for the transient convection-diffusionreaction equation. Comput. Methods Appl. Mech. Engrg. 198 (2009) 2508-2519.

[10] E. Burman and P. Hansbo, Edge stabilization for Galerkin approximations of convection-diffusion-reaction problems. Comput. Methods Appl. Mech. Engrg. 193 (2004) 1437-1453.

[11] E. Burman and G. Smith, Analysis of the space semi-discretized SUPG method for transient convection-diffusion equations. Technical report, University of Sussex (2010).

[12] E. Burman, J. Guzmán and D. Leykekhman, Weighted error estimates of the continuous interior penalty method for singularly perturbed problems. IMA J. Numer. Anal. 29 (2009) 284-314.

[13] B. Cockburn and C.-W. Shu, TVB Runge-Kutta local projection discontinuous Galerkin finite element method for conservation laws. II. General framework. Math. Comp. 52 (1989) 411-435.

[14] R. Codina, Stabilized finite element approximation of transient incompressible flows using orthogonal subscales. Comput. Methods Appl. Mech. Engrg. 191 (2002) 4295-4321.

[15] M. Crouzeix, Une méthode multipas implicite-explicite pour l'approximation des équations d'évolution paraboliques. Numer. Math. 35 (1980) 257-276.

[16] D.A. Di Pietro, A. Ern and J.-L. Guermond, Discontinuous Galerkin methods for anisotropic semidefinite diffusion with advection, SIAM J. Numer. Anal. 46 (2008) 805-831.

[17] A. Ern and J.-L. Guermond, Theory and Practice of Finite Elements, Appl. Math. Sci. 159 (2004).

[18] A. Ern and J.-L. Guermond, Discontinuous Galerkin methods for Friedrichs' systems. I. General theory. SIAM J. Numer. Anal. 44 (2006) 753-778.

[19] J.-L. Guermond, Stabilization of Galerkin approximations of transport equations by subgrid modeling. ESAIM: M2AN 33 (1999) 1293-1316.

[20] J.-L. Guermond, Subgrid stabilization of Galerkin approximations of linear monotone operators. IMA J. Numer. Anal. 21 (2001) 165-197. 
[21] J. Guzmán, Local analysis of discontinuous Galerkin methods applied to singularly perturbed problems. J. Numer. Math. 14 (2006) 41-56.

[22] F. Hecht, O. Pironneau, A. Le Hyaric and J. Morice, FreeFEM++, Version 3.14-0. http://www.freefem.org/ff++/.

[23] C. Johnson, U. Nävert and J. Pitkäranta, Finite element methods for linear hyperbolic problems. Comput. Methods Appl. Mech. Engrg. 45 (1984) 285-312.

[24] C. Johnson and J. Pitkäranta, An analysis of the discontinuous Galerkin method for a scalar hyperbolic equation. Math. Comp. 46 (1986) 1-26.

[25] P. Lesaint and P.-A. Raviart, On a finite element method for solving the neutron transport equation, in Mathematical aspects of Finite Elements in Partial Differential Equations, edited by C. de Boors. Academic Press (1974) 89-123.

[26] D. Levy and E. Tadmor, From semidiscrete to fully discrete: stability of Runge-Kutta schemes by the energy method. SIAM Rev. 40 (1998) 40-73 (electronic).

[27] L. Pareschi and G. Russo, Implicit-explicit Runge-Kutta schemes and applications to hyperbolic systems with relaxation. J. Sci. Comput. 25 (2005) 129-155.

[28] H.-G. Roos, M. Stynes and L. Tobiska, Robust numerical methods for singularly perturbed differential equations, Convectiondiffusion-reaction and flow problems. Springer Series in Computational Mathematics, 2nd edition. Springer-Verlag, Berlin 24 (2008). 